\title{
LA GOBERNANZA DE LA DESCARBONIZACIÓN MARÍTIMA: INICIATIVAS DESDE LOS PUERTOS $^{1}$
}

\section{The Governance of Maritime DeCarbonization: The Initiatives of the Ports}

\author{
Ma REMEDIOS ZAMORA Roselló \\ Profesora contratada-doctora interina \\ Área de Derecho Administrativo (Departamento de Derecho Público) \\ Universidad de Málaga \\ remedios@uma.es
}

Recibido: 27 de marzo de 2018 / Aceptado: 28 de junio de 2018

RESUMEN: El sector portuario se encuentra inmerso en una etapa de transformación marcada por la reducción de emisiones de gases de efecto invernadero, lo que exige la adopción de medidas de eficiencia energética y el desarrollo de combustibles alternativos. Este estudio parte del marco normativo vigente para el control de las emisiones en el transporte marítimo, como referente para el análisis de los puertos, poniendo de relieve el papel de las autoridades portuarias y las iniciativas pioneras que están desarrollando a través de los planes y proyectos de los puertos de Barcelona y Vigo.

RESUM: El sector portuari es troba immers en una etapa de transformació marcada per la reducció d'emissions de gasos d'efecte hivernacle, el que exigeix l'adopció de mesures d'eficiència energètica i el desenvolupament de combustibles alternatius. Aquest estudi parteix del marc normatiu vigent per al control de les emissions en el transport marítim, com a referent per a l'anàlisi dels ports. Posant en relleu el paper de les autoritats portuàries i les iniciatives pioneres que estan desenvolupant, a través dels plans i projectes dels ports de Barcelona i Vigo.

\footnotetext{
${ }^{1}$ Elaborado en el marco del Proyecto de Investigación Ref. DER2017-86637-C3-2-P, financiado por el Ministerio de Economía, Industria y Competitividad, Programa Estatal de Investigación, Desarrollo e Innovación Orientada a los Retos de la Sociedad.
} 


\begin{abstract}
The port sector is in a stage of transformation marked by the reduction of greenhouse gases emissions, which requires the adoption of energy efficiency measures and the development of alternative fuels. This study analyzes the current regulatory framework for the control of emissions in maritime transport, as a reference for the analysis of ports. Emphasizing the role of port authorities and their innovative initiatives, through the plans and projects of the ports of Barcelona and Vigo.
\end{abstract}

PALABRAS CLAVE: Régimen jurídico - Puertos - Transporte marítimo - Cambio climático - Autoridades portuarias.

PARAULES CLAU: Règim jurídic - Ports - Transport marítim - Canvi climàtic Autoritats portuàries.

KEYWORDS: Legal system - Ports - Maritime transport - Climate change - Port authorities.

SUMARIO: I. Introducción. II. La gobernanza internacional: las propuestas de la OMI. III. Más allá de la OMI: la Declaración Tony de Brum. IV. Unión Europea: competitividad, descarbonización y digitalización. V. El sector portuario. 1. Calidad del aire en el ámbito portuario. 2. Medidas de protección ambiental y calidad portuaria de carácter voluntario. 3. Combustibles alternativos: el impulso desde los puertos. 4. Puertos nacionales innovadores: el Port de Barcelona y el Puerto de Vigo. a) El Port de Barcelona y su apuesta por la sostenibilidad económica, social y ambiental. b) El Puerto de Vigo como referente para el crecimiento azul. 5. El papel de las autoridades portuarias en el nuevo desarrollo de los puertos. VI. Conclusiones. VII. Bibliografía.

\title{
I. INTRODUCCIÓN
}

En los últimos años nos encontramos con una creciente atención hacia el sector marítimo; iniciativas como las políticas sobre crecimiento azul de la Unión Europea ponen de relieve la importancia de un sector para el que tradicionalmente no se habían desarrollado políticas transversales. Esta atención también se ha traducido en nuevas propuestas normativas, instrumentos de planificación y proyectos de innovación; y todo ello de la mano de un despertar de las autoridades a las potencialidades que presentan mares y océanos.

La inclusión entre los Objetivos de Desarrollo Sostenible del Objetivo 14, dirigido a "conservar y utilizar en forma sostenible los océanos, los mares y los recursos marinos 
para el desarrollo sostenible", y la celebración de la Conferencia de los Océanos en 2017, la primera Conferencia de Naciones Unidas sobre esta materia, que estuvo dedicada a promover la consecución del citado Objetivo, son ejemplos de este impulso a nivel internacional.

En el ámbito comunitario, el desarrollo de la política marítima integrada de la Unión se ha llevado a cabo a través de una serie de materias transversales que toman como punto de partida el espacio marítimo y la mejora de su gobernanza. En este sentido, cabe destacar las iniciativas sobre ordenación del espacio marítimo y la Directiva Marco sobre la Estrategia Marina, sobre las que volveremos más adelante.

En este estudio vamos a centrarnos en la movilidad; cómo el sector marítimo atraviesa una etapa de transición, al igual que los restantes ámbitos, marcada por la necesidad de reducir su impacto ambiental, con especial atención a la reducción de las emisiones de gases de efecto invernadero y, por ello, a la descarbonización ${ }^{2}$.

El transporte marítimo presenta una gran problemática a la hora de abordar su transformación hacia una economía baja en carbono. En este sentido, nos encontramos con una situación de partida preocupante, ya que es uno de los principales emisores de gases de efecto invernadero; las perspectivas de crecimiento de este sector para las próximas décadas evidencian un incremento paulatino en su contribución al cambio climático si no se adoptan medidas eficaces para alcanzar una eficiencia energética real $^{3}$.

\footnotetext{
${ }^{2}$ Sobre la incidencia del transporte en el cambio climático, BARBERO, J. y RODRÍGUEZ TORNQUIST, R., "Transporte y cambio climático: hacia un desarrollo sostenible y de bajo carbono", Revista Transporte y Territorio, núm. 6, Universidad de Buenos Aires, 2012, pp. 8 a 26.

${ }^{3}$ El Informe de la OMI publicado en 2015, último publicado sobre los gases de efecto invernadero por este organismo, concluye que el transporte marítimo internacional emitió 796 millones de toneladas de $\mathrm{CO}_{2}$ en 2012, lo que supuso una reducción con respecto a los datos de 2007, alcanzando el 2,2\% de las emisiones mundiales de $\mathrm{CO}_{2}$. Sin embargo, las perspectivas de futuro no son positivas; se prevé un incremento considerable de las emisiones en las próximas décadas, que podrá variar en función de las medidas adoptadas en materia energética. INTERNATIONAL MARITIME ORGANIZATION, Third IMO GHC Study 2014, 2015.
}

Según el estudio presentado por la ONG The International Council on Clean Transportation, tras el análisis de las emisiones de gases de efecto invernadero del sector marítimo durante el período 20132015, si bien se mejoró la eficiencia de los buques, no se consiguió reducir el valor absoluto de las emisiones. Se produjo un incremento de las emisiones de $\mathrm{CO}_{2}$, puesto que el aumento en las distancias recorridas, ante la mayor demanda de este tipo de transportes, neutralizó los efectos positivos de las medidas de eficiencia energética. OLMER, N., COMER, B., ROY, B., MAO, X. y RUTHERFORD, D., Greenhouse gas emissions from global shipping, 2013-2015, The International Council on Clean Transportation, octubre 2017. El texto completo del informe se encuentra disponible en 
A todo ello cabe añadir que el nivel de desarrollo tecnológico para los próximos años supondrá una transformación del modelo actual del transporte marítimo. En la actualidad, ya se ha diseñado el primer buque portacontenedores del mundo completamente eléctrico, por lo que no genera emisiones y, además, es autónomo; el Yara Birkeland empezará a probarse en 2019 y se espera que pueda realizar operaciones autónomas para 2020.

El análisis del transporte marítimo desemboca en el sector portuario como nudo de comunicaciones, origen y fin de este sistema de movilidad y central logística para la recepción de pasajeros y la distribución de mercancías. La reflexión sobre las políticas portuarias, las iniciativas del sector y los proyectos concretos que se están desarrollando en algunos puertos españoles también nos ofrecen la posibilidad de conocer localmente cómo se están aplicando las iniciativas internacionales por parte de las autoridades competentes.

El estudio sobre la respuesta de los organismos, las instituciones y las administraciones sobre las necesidades del sector es la esencia para conocer en qué medida la industria marítima se adaptará realmente al cumplimiento de los objetivos ambientales que debe asumir.

\section{LA GOBERNANZA INTERNACIONAL: LAS PROPUESTAS DE LA OMI}

La principal característica del transporte marítimo es su vocación internacional; por lo tanto, resultan determinantes los instrumentos que superan la esfera regional y la nacional. Sin embargo, en el Acuerdo de París de 2015 se excluyó el transporte marítimo, al igual que la aviación, de su ámbito de aplicación ${ }^{4}$. Por ello, la labor de la

\footnotetext{
<https://www.theicct.org/publications/GHG-emissions-global-shipping-2013-2015>.

${ }^{4}$ En relación con las iniciativas internacionales de lucha contra el cambio climático y su evolución en los últimos años, BELLEVER CAPELLA, V., "El derecho al cambio climático: ascenso y decadencia del Protocolo de Kioto", Fernández Ruiz-Gálvez, E. (coord.), El futuro de los derechos humanos, Tirant lo Blanch, Valencia, 2016, pp. 208-237; SARASÍBAR IRIARTE, M., "La Cumbre del Clima de París: ¿un avance importante en la política del cambio climático?", Administración \& cidadanía: revista da Escola Galega de Administración Pública, vol. 11, núm. 2, 2016, pp. 307-314; CAMPINS ERITJA, M., "Entre la urgència a Fiji i la complexitat a Bonn. El lent camí per a la posada en marxa de l'Acord de París", Revista Catalana de Dret Ambiental, vol. VIII, núm. 2, 2017, pp. 1-8. Sobre la responsabilidad estatal respecto al fenómeno del cambio climático, RODRÍGUEZ GARCÍA, N., "Responsabilidad del Estado y cambio climático: El caso Urgenda contra Países Bajos", Revista Catalana de Dret Ambiental, vol. VII, núm. 2, 2016, pp. 1-38; LAGO CANDEIRA, A., "El avance del Protocolo de Montreal hacia la mitigación del cambio climático (Enmienda de Kigali)", en López Ramón, F., Observatorio de Políticas Ambientales 2017, CIEMAT, Madrid, 2017, p. 39 y ss.
} 
Organización Marítima Internacional (OMI) se ha convertido en la vía para alcanzar una transformación energética en este ámbito.

A este respecto, debemos resaltar las reticencias de algunos sectores a incluir las medidas sobre control de emisiones del sector marítimo en los debates y acuerdos globales sobre cambio climático. En concreto, el propio secretario de la OMI se manifestó contrario a debatir estas medidas en cualquier otro foro, aludiendo a la gran repercusión sobre la economía mundial del sector marítimo y, por lo tanto, a las graves consecuencias que pudieran originarse de las decisiones adoptadas por los Estados al margen de la Organización ${ }^{5}$.

La labor desarrollada por la OMI se ha plasmado en propuestas relevantes que han impulsado la eficiencia energética de los buques, haciendo hincapié en los requisitos mínimos sobre las emisiones de dióxido de carbono y en la mejora de la gestión ambiental del transporte marítimo a través del análisis y la evaluación de los datos suministrados por los propios buques ${ }^{6}$.

Una de las principales iniciativas que se están desarrollando en la actualidad es el Proyecto de Asociaciones para la Eficiencia Energética Marítima Mundial (GloMEEP), auspiciado por la OMI, el Programa de las Naciones Unidas para el Desarrollo (PNUD) y el Fondo para el Medio Ambiente Mundial (FMAM), cuya finalidad es la adopción e implantación de medidas para la eficiencia energética en el sector marítimo. Actualmente son diez países los que están desarrollando esta iniciativa piloto: Argentina, China, Filipinas, Georgia, India, Jamaica, Malasia, Marruecos, Panamá y Sudáfrica. El objetivo último es la reducción de las emisiones de gases de efecto

\footnotetext{
${ }^{5}$ El entonces secretario de la OMI, Koji Sekimizu, se expresó en los siguientes términos en septiembre de 2015: "In the process leading up to the Paris meeting, world leaders might be tempted to consider specific measures aimed at reducing shipping's overall contribution of $\mathrm{CO}_{2}$ emissions, such as an overall cap. Such measures would artificially limit the ability of shipping to meet the demand created by the world economy, or would unbalance the level playing field that the shipping industry needs for efficient operation, and therefore must be avoided. In addition, fiscal measures such as a levy on fuel are under active consideration, but such measures require careful analysis and development, considering a host of environmental, technical, economic and geopolitical factors. For these matters, so crucial to the world's future, there are no simple answers. But IMO is the most capable and appropriate forum for such complex considerations to occur and be resolved". SEKIMIZU, K., Shipping and climate change, Statement from IMO Secretary-General, septiembre 2015, disponible en $<$ http://www.imo.org/en/MediaCentre/HotTopics/GHG/Documents/Shipping\%20and\%20climate\%20chan ge.pdf $>$.

${ }^{6}$ Desde el 1 de enero de 2013 se encuentran en vigor las enmiendas al anexo VI del Convenio MARPOL como nuevo capítulo bajo el título "Reglas sobre la eficiencia energética de los buques".
} 
invernadero originadas en los buques; para ello, se fomentan las sinergias entre los Estados mediante las asociaciones mundiales y regionales, a la vez que se impulsa la adopción de medidas a nivel nacional. La consecución de estos fines se lleva a cabo a través de la discusión y el debate sobre las propuestas de reformas legales, políticas e institucionales, las actividades de sensibilización y capacitación, y el establecimiento de asociaciones público-privadas para alentar la transferencia tecnológica ${ }^{7}$.

Otro de los grandes proyectos de la OMI es la Red Mundial de Centros de Cooperación de Tecnología Marítima (MTCC), financiada por la Unión Europea. Se inauguró oficialmente en diciembre de 2017 y está constituida por los siguientes centros:

- MTCC-América Latina, con sede en la Universidad Marítima Internacional de Panamá (Panamá).

- MTCC-África, albergado por la Universidad de Agricultura y Tecnología Jomo Kenyatta (Kenia).

- MTCC-Asia, ubicado la Universidad Marítima de Shanghái (China).

- MTCC-Caribe, radicado en la Universidad de Trinidad y Tobago (Trinidad y Tobago).

- MTCC-Pacífico, albergado por la Comunidad del Pacífico (SPC), Suva (Fiji).

La labor de estos centros se va a centrar en la reducción de emisiones con origen en los buques sobre la base de la aplicación de las innovaciones tecnológicas y la mejora de la eficiencia energética en el sector marítimo. Su ubicación en África, Asia, el Caribe, América Latina y el Pacífico responde a la necesidad de impulsar en los países del entorno el desarrollo de políticas marítimas nacionales, así como la implementación de sistemas de compilación y notificación de datos de carácter voluntario, el desarrollo de

\footnotetext{
${ }^{7}$ La viabilidad económica de las medidas para controlar las emisiones es una realidad que se ha puesto de manifiesto por algunos autores. En este sentido, WAN, Z., MAKHLOUFI, A., CHEN, Y. y TANG, J., "Decarbonizing the international shipping industry: Solutions and policy recommendations", Marine Pollution Bulletin, vol. 126, enero 2018, p. 435: "Current and possible future mitigation strategies commendably include transdisciplinary solutions covering technical, operational, and market aspects; however, widespread application requires them to be evidence-based and economically viable. Their success also hinges on the continuous assessment and refinement in real-world applications. Synergies, led by the top maritime commander, the IMO, must speed up cooperation in the global shipping community, linking scientists, engineers, businesses, and policymakers to honor the centuries-old industry in the 'spirit of Paris"'. Sobre la adopción de medidas de mercado, SHI, Y., "Reducing greenhouse gas emissions from international shipping: Is it tima to consider market-based measures?", Marine Policy, vol. 64, febrero 2016, pp. 123-134.
} 
medidas de eficiencia energética y la aplicación de las novedades tecnológicas adecuadas para conseguir una reducción significativa en las emisiones de carbono en el transporte marítimo. Los destinatarios de esta iniciativa son los países en desarrollo, con especial atención a aquellos que se encuentran en una situación más precaria desde el punto de vista de la implantación de los avances tecnológicos, así como los Estados insulares en desarrollo.

En 2017 se adoptó por el Comité de Protección del Medio Marino de la OMI un esquema de siete pasos que han de ser desarrollados mediante un programa interno durante 2018 y que aspiran a descarbonizar el transporte marítimo ${ }^{8}$. En el seno de este comité se ha desarrollado un importante debate en el que se planteó la posibilidad de establecer una serie de objetivos que siguieran los principios del Acuerdo de París. No obstante, el consenso es difícil. Por una parte, nos encontramos con un conjunto de Estados que se encuentran directamente afectados por el cambio climático y que exigen la adopción de medidas urgentes, principalmente los países isleños del Pacífico. Mientras que, por otra, los países productores de petróleo como Brasil o Arabia Saudí se muestran reacios y ponen de manifiesto los impactos negativos que estas medidas reductoras podrían ocasionar ${ }^{9}$.

El objetivo era que la OMI adoptara una estrategia para concretar medidas, plazos y objetivos a cumplir a la mayor brevedad a fin de poder ofrecer los datos necesarios sobre la contribución inicial del sector marítimo para la reducción de emisiones de $\mathrm{CO}_{2}$ en el proceso de evaluación de 2018 en el marco del Acuerdo de París ${ }^{10}$.

\footnotetext{
${ }^{8}$ La estrategia inicial va a incluir: 1. Preámbulo/introducción/contexto, incluidas las hipótesis referidas a las emisiones; 2. Visión; 3. Niveles de ambición; 4. Lista de posibles medidas adicionales a corto, medio y largo plazo, con los plazos posibles y sus repercusiones para los Estados; 5. Obstáculos y medidas de apoyo; creación de capacidad y cooperación técnica; I+D; 6 . Medidas de seguimiento para la elaboración de la estrategia revisada; 7. Examen periódico de la estrategia. Disponible en: <http://www.imo.org/es/MediaCentre/MeetingSummaries/MEPC/Paginas/MEPC-71.aspx>.

${ }^{9}$ Sobre las medidas adoptadas en el seno del Comité de Protección del Medio Marino durante 2017, HOFFMANN, J. (coord.), Review of Maritime Transport 2017, United Nations, UNCTAD/RMT/2017, 2017, pp. 89 y 90. Disponible en: 〈http://unctad.org/en/PublicationsLibrary/rmt2017_en.pdf〉.

${ }^{10}$ La Unión Europea se ha posicionado apoyando el debate que existe en la OMI sobre la reducción de las emisiones del transporte marítimo internacional. A este respecto, COMISIÓN EUROPEA, Informe al Parlamento Europeo y al Consejo. "Dos años después de París - Avances hacia el cumplimiento de los compromisos climáticos de la UE”, $\operatorname{COM(2017)~} 646$ final/2, Bruselas, 27 de noviembre de 2017.
}

Desde el ámbito del sector privado, también podemos destacar la valoración que ha realizado la Cámara Naviera Internacional (ICS) sobre la labor de la OMI en este ámbito. Estas referencias son de interés, puesto que la ICS representa a las asociaciones de navieros nacionales de todo el mundo, responsables de 
En abril de 2018, durante el 72..$^{\circ}$ período de sesiones del Comité de Protección del Medio Marino, a la que asistieron representantes de más de cien Estados, se adoptó la estrategia inicial para la reducción de gases de efecto invernadero de los buques. En esta estrategia se incluye un itinerario de reducción de emisiones de dióxido de carbono en consonancia con los objetivos de temperatura del Acuerdo de París, se definen las líneas de actuación por las que han de regirse los Estados miembros y se especifican los niveles de ambición para reducir las emisiones de efecto invernadero y los principios rectores.

La estrategia refuerza el compromiso de la OMI en la reducción de las emisiones de gases de efecto invernadero procedentes del transporte marítimo internacional, y establece la urgencia de su eliminación gradual. No obstante, se reconoce que la eliminación gradual se llevará a cabo en este siglo, lo que implica un ámbito temporal poco exigente para la adopción de medidas de carácter urgente.

Entre los principales acuerdos se encuentra la reducción en el total de las emisiones de gases de efecto invernadero provocadas por el transporte marítimo internacional de, al menos, el 50\% para el año 2050 (en comparación con los niveles de 2008). También se incluyen en el texto un listado de posibles medidas adicionales, plazos de aplicación y repercusiones para los Estados.

La estrategia no satisface las expectativas que se habían generado ${ }^{11}$, y la presión de los Estados contrarios a un endurecimiento en la política de emisiones de gases de efecto invernadero ha bloqueado la adopción de acuerdos más ambiciosos que permitirían la

más del $80 \%$ de la capacidad de transporte de la flota mercante mundial. En su informe anual se ha pronunciado en los siguientes términos, p. 9: "ICS is confident that IMO can adopt an ambitious strategy by 2018 matching the ambition of the Paris Agreement. However, ICS members have concluded that to be consistent with the spirit of the Paris Agreement, IMO needs to agree a baseline year for peak CO2 emissions from shipping, as well as some serious long term aspirations to dramatically cut the sector's total CO2. ICS also believes that IMO should adopt aspirational objectives for the sector as a whole, rather than set targets for individual ships, in the same way that governments have already agreed $\mathrm{CO} 2$ commitments for their national economies under the Paris Agreement. But IMO also needs to agree measures for delivery which ICS would like to see in place by 2023 (when UNFCCC will be conducting a major global stocktaking exercise of progress towards holding global temperature increases to below two degrees higher than pre-industrial levels)". INTERNATIONAL CHAMBER OF SHIPPING, 2017 Annual Review, ICS, 2018, texto completo disponible en <http://www.ics-shipping.org/docs/default-source/ICSAnnual-Review-2017/ics-annual-review-2017.pdf?sfvrsn=10>

${ }^{11}$ En representación de la Unión Europea, los comisarios Bulc y Arias Cañete han manifestado su satisfacción por la adopción del acuerdo, pero no han ocultado que las propuestas de la Unión eran más ambiciosas y que lo consideran un primer paso que ha de ser objeto de revisiones y mejoras para alcanzar fines más comprometidos con la política de cambio climático. Texto completo disponible en $<$ https://ec.europa.eu/transport/modes/maritime/news/2018-04-13-imo-agreement-co2_sv?2ndlanguage $=\mathrm{en}>$ 
obtención de resultados con mayor rapidez. Por lo tanto, se ha de continuar en esta línea, que permite una inclusión paulatina del sector marítimo en la política global contra el cambio climático, si bien se evidencia que sigue lastrado en relación con otros sectores y presenta déficits, subsanables pero determinantes, para que la contribución del ámbito marítimo al calentamiento global pueda ser superada.

Este acuerdo inicial será objeto de revisión en 2023, por lo que las miras deben ponerse en la consecución de un mayor compromiso. El transporte marítimo no puede mantener su estatus excepcional mientras el resto de ámbitos adquiere compromisos más amplios para la reducción de las emisiones de gases de efecto invernadero.

\section{MÁS ALLÁ DE LA OMI: LA DECLARACIÓN TONY DE BRUM}

Los datos sobre el incremento en el transporte marítimo y el aumento global de emisiones, a pesar de las medidas de eficiencia y control instauradas desde la OMI, ponen de manifiesto que no se está avanzando en este sector con la debida diligencia.

Como ya hemos comentado, la exclusión del transporte marítimo del Acuerdo de París no puede ser un obstáculo para que este sector no aspire al cumplimiento de los requisitos mínimos establecidos para reducir las emisiones de gases de efecto invernadero y controlar de esta forma el paulatino incremento de la temperatura global.

Esta alteración, que está afectando a todo el planeta, es todavía más grave en aquellos Estados que están en riesgo de desaparecer por la subida del nivel del mar, que lógicamente se encuentran más sensibilizados para reducir los efectos del cambio climático y tratan de presionar a la comunidad internacional para que adopte medidas reales y efectivas.

Uno de los líderes de las Islas Marshall, situadas en el Pacífico, Tony de Brum, es quien da nombre a la más reciente de estas iniciativas dirigidas a implantar medidas en el ámbito marítimo ${ }^{12}$. La Declaración Tony de Brum fue adoptada en la cumbre One Planet

\footnotetext{
12 Tony de Brum fue embajador contra el cambio climático de las Islas Marshall y Premio Nobel Alternativo. Su trayectoria política estuvo marcada por su lucha contra el cambio climático y las armas nucleares. Durante las negociaciones del Acuerdo de París se convirtió en el abanderado de la "coalición de la gran ambición", que instó a limitar el aumento de la temperatura a 1,5 grados con respecto a los niveles preindustriales.
} 
Summit ${ }^{13}$, celebrada en Francia con motivo del segundo aniversario del Acuerdo de París y en la que se reunieron representantes del sector público y el privado, así como filántropos, para tratar de impulsar la lucha contra el cambio climático.

Este texto es una llamada al cumplimiento de los objetivos de lucha contra el cambio climático y, en especial, del compromiso para controlar la subida de la temperatura del planeta. No obstante, se centra en la reducción de emisiones en el sector marítimo internacional y realiza un llamamiento a la adopción de medidas urgentes, tanto en el transporte marítimo internacional como en todos los demás sectores de la actividad humana, para el futuro del planeta y de la humanidad. La finalidad última es el establecimiento de valores límite de emisión a corto plazo para llegar a la neutralidad a mediados de siglo.

Por ello, se reconocen los esfuerzos de la OMI y se insta al cumplimiento de la hoja de ruta establecida para los próximos años. En este documento se hace hincapié en el establecimiento de directrices para el sector marítimo que sigan la línea de las adoptadas en el Acuerdo de París y en la importancia de no crear distorsiones en la competencia, por lo que las disposiciones deberían aplicarse por igual a todos los buques independientemente de su bandera.

Asimismo, en esta declaración se hace un especial llamamiento al estudio de las medidas adoptadas para los Estados en desarrollo y los Estados insulares en desarrollo por sus singulares características y el mayor impacto que pueden generar las futuras propuestas sobre estos territorios.

Desde la Unión Europea también se han asumido los objetivos de esta declaración y se ha instado a la OMI a adoptar medidas adicionales para que la contribución del transporte marítimo a la lucha contra el cambio climático sea coherente con los objetivos del Acuerdo de París, a implantar medidas a corto, medio y largo plazo, y a adoptar en 2018 una estrategia inicial para la reducción de las emisiones de gases de efecto invernadero ${ }^{14}$.

\footnotetext{
${ }^{13}$ El 12 de diciembre de 2017 se celebró esta cumbre, donde se presentaron treinta proyectos para luchar contra el cambio climático.

${ }^{14}$ COUNCIL OF THE EUROPEAN UNION, Council Conclusions on Climate Diplomacy, Bruselas, 26 de febrero de 2018, disponible en: <http://data.consilium.europa.eu/doc/document/ST-6125-2018INIT/en/pdf $>$.
} 


\section{UNIÓN EUROPEA: COMPETITIVIDAD, DESCARBONIZACIÓN Y DIGITALIZACIÓN}

El compromiso de la Unión Europea en la lucha contra el cambio climático es una realidad que se ha puesto de manifiesto en numerosas iniciativas implantadas en la última década. A este respecto, cabe destacar el Paquete Europeo de Energía y Cambio Climático 2013-2020, adoptado en 2008. Estas iniciativas han tenido su continuación en las propuestas incluidas en el Marco de Políticas de Energía y Cambio Climático 20212030, que aumenta los umbrales a alcanzar imponiendo una reducción de las emisiones de gases de efecto invernadero del $40 \%$ sobre las emisiones de 1990, un $27 \%$ de mejora en la eficiencia energética y un incremento en el consumo de energía renovable que permita llegar al $27 \%$ del total ${ }^{15}$.

Estas propuestas deben relacionarse con las iniciativas específicas que se están desarrollando en el ámbito marítimo. En 2017 se celebró el 10. ${ }^{\circ}$ aniversario de la política marítima integrada de la Unión Europea y, desde el año 2012, la Unión ha estado desarrollando iniciativas sobre crecimiento azul, con la finalidad última de aprovechar el potencial de océanos, mares y costas para el crecimiento económico y la creación de empleo. Este crecimiento azul se basa en cinco pilares fundamentales: la energía azul, la acuicultura, el turismo marítimo, costero y de cruceros, los recursos minerales marinos y la biotecnología azul.

Entre las iniciativas de la política marítima integrada de la Unión cabe destacar la ordenación del espacio marítimo, que toma como referencia la Directiva adoptada en 2014, mediante la cual se aspira al crecimiento sostenible de las economías marítimas, al desarrollo sostenible de los espacios marinos y al aprovechamiento sostenible de los recursos marinos ${ }^{16}$. En esta disposición se define la ordenación del espacio marítimo

\footnotetext{
15 SARASÍBAR IRIARTE, M., "Cambio climático: su regulación jurídica como grave problema ambiental”, López Ramón, F., Observatorio de Políticas Ambientales 2017, CIEMAT, Madrid, 2017, pp. 882 y ss; SARASÍBAR IRIARTE, M., "Energías renovables y cambio climático: un binomio condenado a entenderse", Galán Vioque, R. y González Ríos, I. (dirs.), Derecho de las energías renovables y la eficiencia energética en el horizonte 2020, Aranzadi, Cizur Menor (Navarra), 2017, p. 515 y ss.

${ }^{16}$ Directiva 2014/89/UE del Parlamento Europeo y del Consejo, de 23 de julio de 2014, por la que se establece un marco para la ordenación del espacio marítimo (DO L 257, de 28 de agosto de 2014). Esta disposición ha de completarse en nuestro país con la referencia al Real Decreto 363/2017, de 8 de abril, por el que se establece un marco para la ordenación del espacio marítimo, BOE, núm. 86, de 11 de abril de 2017. En esta disposición se regula el contenido de los planes de ordenación del espacio marítimo, que determinarán la distribución espacial y temporal de las distintas actividades, usos e intereses que se han de desarrollar en este espacio; entre ellos se encuentran las rutas de transporte marítimo y el tráfico
} 
como "el proceso mediante el cual las autoridades competentes del Estado miembro analizan y organizan las actividades humanas en las zonas marinas con el fin de alcanzar los objetivos ecológicos, económicos y sociales"; en definitiva, identificar los usos del mar y fomentar una gobernanza adecuada de este espacio. Asimismo, estos planes tendrán en cuenta las interacciones entre tierra y mar, y determinarán las rutas de transporte marítimo y el tráfico marítimo ${ }^{17}$.

Asimismo, debemos mencionar la Directiva Marco sobre la Estrategia Marina, cuyo objetivo es la consecución o mantenimiento de un buen estado ambiental del medio marino; a este fin incluye un conjunto de medidas de planificación, conservación, protección y mejora ${ }^{18}$. Entre estas medidas destacan las estrategias marinas, instrumento de planificación clave de cada demarcación marina que se configura como el marco de referencia para las políticas sectoriales y actuaciones administrativas que incidan en el medio marino. Sus objetivos específicos se centran en proteger y preservar el medio marino, así como en recuperar los ecosistemas marinos que se encuentren afectados negativamente, en prevenir y reducir los vertidos, y en garantizar que las actividades y los usos en el medio marino sean compatibles con la preservación de su biodiversidad.

En marzo de 2017, los ministros europeos de Transportes adoptaron la denominada Declaración de La Valeta, que define las directrices de la política comunitaria sobre el transporte marítimo ${ }^{19}$. Los tres principios rectores de la política de transporte marítimo de la UE hasta 2020 son la competitividad, la descarbonización y la digitalización. La

marítimo, los espacios protegidos, las zonas de pesca y acuicultura, y las actividades turísticas, entre otros (art. 10).

${ }^{17}$ A este respecto, FUENTES GASÓ, R., “Avances en la protección y conservación del medio marino español. El nuevo marco para la ordenación del espacio marítimo", Revista Catalana de Dret Ambiental, vol. VIII, núm. 1, 2017, pp. 1-44; LOZANO CUTANDA, B., "Real Decreto 363/2017, de 8 de abril: la ordenación de los distintos usos y de las actividades económicas en el espacio marítimo", Diario La Ley, núm. 8999, 2017; SANZ LARRUGA, J., "La nueva ordenación del espacio marítimo. Análisis del Real Decreto 363/2017, de 8 de abril", Práctica urbanística. Revista mensual de urbanismo, núm. 150, 2018.

${ }^{18}$ La norma comunitaria afirma que el buen estado ambiental del medio marino se caracteriza por “océanos y mares ecológicamente diversos y dinámicos, limpios, sanos y productivos en el contexto de sus condiciones intrínsecas, y en el que la utilización del medio marino sea sostenible, quedando así protegido su potencial de usos, actividades y recursos por parte de las generaciones actuales y futuras". Directiva 2008/56/CE del Parlamento Europeo y del Consejo, de 17 de junio de 2008, por la que se establece un marco de acción comunitaria para la política del medio marino (DO L 146, de 25 de junio de 2008). Esta norma se transpuso en nuestro ordenamiento interno mediante la Ley 41/2010.

19 Ministerial Declaration on Priorities for the EU's maritime transport policy until 2020: Competitiveness, Decarbonisation, Digitalisation to ensure global connectivity, and efficient internal market and a world-class maritime cluster (Valletta Declaration), 29 de marzo de 2017. Disponible en: <https://www.eu2017.mt/en/Documents/Valletta_Declaration_on_the_EU_maritime_transport_policy.pdf $>$. 
doble finalidad de esta política es mantener y fomentar este medio de transporte para las mercancías y personas, y lograr una reducción de su impacto sobre el medio ambiente.

Entre las recomendaciones que se incluyen en la Declaración cabe destacar las referencias al compromiso comunitario para la reducción de las emisiones con origen en este medio de transporte. A nivel internacional se realiza un llamamiento, tanto a los Estados como a la Comisión, para que en 2018 impulsen la adopción de una estrategia inicial de la OMI que sea ambiciosa y para que empleen los medios técnicos y humanos necesarios para implementar esta iniciativa, que también deberá acompañarse de un incremento en las inversiones y en la investigación a fin de conseguir la reducción en las emisiones de gases $^{20}$.

También se insta a la Comisión a que proponga las modificaciones necesarias en el Reglamento comunitario sobre seguimiento, verificación y notificación de buques ${ }^{21}$ a fin de lograr su armonización con el sistema mundial de recopilación de datos sobre emisiones de dióxido de carbono, cuyo marco jurídico está previsto que sea aprobado en breve.

En lo que respecta al uso de energías y combustibles alternativos en los buques, los esfuerzos se centran en el impulso al gas natural licuado (GNL) y en la armonización de normas sobre repostaje en materia de conectores y procedimientos.

A nivel regional, y con la finalidad última de la aplicación efectiva de las medidas adoptadas en la Unión Europea, también se hace un llamamiento para que la Comisión y los Estados miembros cooperen para conseguir el cumplimiento efectivo de la normativa vigente sobre emisiones procedentes de buques, con especial atención al intercambio de datos.

Además de estas previsiones específicas sobre las emisiones del sector marítimo, se incluyen otras propuestas de gran interés que se centran en el empleo, las ayudas y la

\footnotetext{
${ }^{20}$ Sobre las iniciativas adoptadas por la Unión Europea para reducir las emisiones con origen en el transporte marítimo, MARTÍNEZ PÉREZ, E., "Mercado de emisiones y transporte internacional: acciones unilaterales e integración de la navegación aérea y el transporte marítimo en el ETS", Sanz Rubiales, I. (coord.), Cambio climático y Unión Europea. Presente y futuro del mercado europeo de emisiones, Tirant lo Blanch, Valencia, 2014, p. 309 y ss.

${ }^{21}$ Reglamento (UE) 2015/757 del Parlamento Europeo y del Consejo, de 29 de abril de 2015, relativo al seguimiento, notificación y verificación de las emisiones de dióxido de carbono generadas por el transporte marítimo y por el que se modifica la Directiva 2009/16/CE, DOUE L123/55, de 19 de mayo de 2015.
} 
reducción de las trabas administrativas. En concreto, la Declaración apuesta por el desarrollo de iniciativas y políticas comunitarias que promuevan el empleo de la gente de mar; para ello, considera esencial el establecimiento de unas condiciones sociales dignas y de calidad, y la adopción de medidas que hagan más atractivo el sector para los jóvenes. Asimismo, también se recuerdan la brecha de género existente en este sector y la importancia de fomentar el empleo entre las mujeres, así como su participación.

El objetivo de competitividad se fortalece al situarlo como una prioridad clave que se sustenta sobre las directrices comunitarias en materia de ayudas de Estado al transporte marítimo, que permiten mantener la competitividad del sector marítimo comunitario a escala global. Todo ello, a la vez que se fomentan la calidad y el atractivo de las banderas de los Estados comunitarios.

Si el sector marítimo ha de ocupar una posición preferente en la economía comunitaria, es necesario reducir las barreras y mejorar su eficiencia. La revisión de las formalidades administrativas, la introducción del manifiesto electrónico y el impulso a la "ventanilla única marítima europea" son algunas de las iniciativas dirigidas al cumplimiento de los objetivos de competitividad y digitalización. En esta declaración se reconoce de forma expresa que la principal preocupación de todos los sujetos públicos y privados que integran la industria y el transporte marítimo es la simplificación administrativa y la configuración de un sistema de intercambio de información digital integral e interoperable.

La cuestión que debemos plantearnos es cómo ha evolucionado la política marítima comunitaria durante esta década y, en particular, cuáles son las principales diferencias que encontramos entre la Declaración de La Valeta y la Declaración de Limasol ${ }^{22}$.

A este respecto, cabe recordar que, tras la publicación de la Comunicación de la Comisión sobre crecimiento azul, la reunión de los ministros europeos en Limasol dio como resultado una declaración en la que se ponía el acento sobre el crecimiento, la competitividad y el empleo en el sector marítimo. El marco de aplicación de este texto no solo comprendía el transporte marítimo, sino que también se pronunciaba sobre un

\footnotetext{
${ }^{22}$ Declaración de los Ministros europeos responsables de la Política Marítima Integrada y la Comisión Europea sobre una Agenda Marina y Marítima para el crecimiento y el empleo, "Declaración de Limasol", Limasol, 8 de octubre de 2012.
} 
modelo completo de economía azul sostenible y la determinación de una hoja de ruta trazada a través de la Agenda Marina y Marítima Europea.

Son numerosos los elementos sobre los que existe una continuidad; de hecho, se repiten argumentos como la necesidad de reducir la carga administrativa, impulsar el empleo y hacer frente a los desafíos globales que, de forma más pronunciada que en otros ámbitos, caracterizan al sector marítimo. En definitiva, buena parte de los objetivos de hace un lustro aún no se han logrado, por lo que continúan entre las prioridades de las instituciones comunitarias, sin que los avances hayan permitido superar estas debilidades.

\section{EL SECTOR PORTUARIO}

Los puertos se configuran como un elemento clave para el desarrollo de una economía azul que pueda definirse como sostenible desde la perspectiva medioambiental. En la ya analizada Declaración de La Valeta se recuerda la necesidad de priorizar la actuación sobre los puertos como instrumento para hacer frente a los retos económicos, ambientales y sociales que presenta el transporte marítimo, ya que en los puertos es donde se generan valores y empleos en todas las zonas costeras del entorno comunitario, y también donde en mayor medida se sufren los impactos ambientales de este modelo de transporte ${ }^{23}$.

Los puertos están conectados con toda la economía azul y superan la esfera del transporte marítimo; la pesca, el turismo y las relaciones culturales y sociales son solo algunos de los ejemplos de la relevancia del sector portuario en el ámbito marítimo.

En las últimas décadas la globalización y el incremento del tráfico mundial de mercancías han generado un resurgimiento del sector portuario. Los puertos han aumentado considerablemente su número de trabajadores, sus infraestructuras y su impacto ambiental, territorial y socioeconómico, proyectándose sobre las localidades en las que se ubican y marcando su desarrollo y modelo de crecimiento.

Este contexto exige una respuesta que se manifieste a través de políticas portuarias que sean capaces de realizar un diagnóstico de las necesidades actuales, así como de las

\footnotetext{
${ }^{23}$ Sobre las iniciativas comunitarias en materia portuaria, EGUINOA DE SAN ROMÁN, R., Derecho comunitario y puertos de interés general, Atelier Libros, Barcelona, 2017.
} 
venideras a medio y largo plazo. Se ha de proyectar sobre las Administraciones públicas la obligación de adoptar las medidas de planificación que permitan hacer frente a estos desafíos. De esta forma, se fomentarán e incentivarán aquellos modelos de desarrollo que sean positivos para el sector y que pongan en valor las demandas sociales, ambientales y económicas ${ }^{24}$.

En el ámbito financiero, ya se encuentra fijado un marco para la prestación de servicios portuarios y normas comunes sobre la transparencia de los puertos, adoptado mediante Reglamento comunitari ${ }^{25}$. Asimismo, debemos destacar los instrumentos de planificación del medio marino impulsados desde la Unión Europea y ya implantados en nuestro ordenamiento interno. Los planes de ordenación del espacio marítimo tienen como finalidad última el fomento del crecimiento sostenible de las economías marítimas, el desarrollo sostenible de los espacios marinos y el aprovechamiento sostenible de los recursos marinos. Asimismo, estos planes tendrán en cuenta las interacciones entre tierra y mar, y determinarán las rutas de transporte marítimo y el tráfico marítimo ${ }^{26}$.

La adopción de medidas que garanticen la descarbonización del sector marítimo pasa inexorablemente por la transformación de los puertos, elementos básicos para la interconexión mar-tierra y piezas determinantes en la cadena logística de la industria marítima $^{27}$.

\footnotetext{
${ }^{24}$ DOERR, O., Políticas portuarias, Naciones Unidas, Santiago de Chile, 2011, p. 7 y ss.

${ }^{25}$ Reglamento (UE) 2017/352 del Parlamento Europeo y del Consejo, de 15 de febrero de 2017, por el que se crea un marco para la prestación de servicios portuarios y se adoptan normas comunes sobre la transparencia financiera de los puertos, DO L 57, de 3 de marzo de 2017. Como se señala en el considerando primero de este texto: "La plena integración de los puertos en unas cadenas logísticas y de transporte sin discontinuidades es necesaria para contribuir al crecimiento y a un uso y un funcionamiento más eficientes de la red transeuropea de transportes y del mercado interior. Ello requiere unos servicios portuarios modernos que contribuyan al uso eficiente de los puertos y a un clima favorable para la inversión, con vistas a desarrollar puertos acordes con los requisitos actuales y futuros en materia de transporte y logística".

${ }^{26}$ Real Decreto 363/2017, de 8 de abril, por el que se establece el marco para la ordenación del espacio marítimo (BOE, núm. 86, de 11 de abril de 2017). A este respecto, FUENTES GASÓ, R., "Avances en la protección y conservación del medio marino español. El nuevo marco para la ordenación del espacio marítimo", Revista Catalana de Dret Ambiental, vol. VIII, núm. 1, 2017, pp. 1-44; SANZ LARRUGA, J., "La nueva ordenación del espacio marítimo. Análisis del Real Decreto 363/2017, de 8 de abril", Práctica urbanística. Revista mensual de urbanismo, núm. 150, 2018.

${ }^{27}$ En relación con las medidas adoptadas en España sobre cambio climático, SARASÍBAR IRIARTE, M., "Políticas y medidas de mitigación del cambio climático en el ámbito jurídico español", El cambio climático: Una mirada desde Cataluña. Primer Congreso Catalán de Derecho Ambiental, Tarragona, 1719 de enero de 2018, <http://wwwa.fundacio.urv.cat/congressos/congres-catala-dretambiental/presentaciones>. Sobre la experiencia de algunos puertos europeos y norteamericanos que han
} 
Asimismo, las innovaciones técnicas van a condicionar el sistema portuario para los próximos años, de ahí que algunos puertos ya se estén preparando para hacer frente a una renovación como la que supondrá la puesta en servicio de buques total o parcialmente autónomos. A nivel nacional, podemos destacar el caso del puerto de Bilbao, que ha apostado por la digitalización y que espera convertirse en el mejor puerto equipado para recibir este tipo de buques, que permiten reducir los costes y las emisiones y, a la vez, mejorar la competitividad.

En el caso de los puertos españoles, cabe destacar dos elementos de gran interés: por una parte, la posición estratégica de nuestro país para el transporte marítimo y, por otra, la calidad de las infraestructuras portuarias. Respecto al primer elemento, poco hay que añadir, tan solo que no es suficiente con disponer de una posición geográfica clave, sino que es necesario saber aprovechar estas cualidades intrínsecas al sector portuario español y ponerlas en valor frente a otros Estados que puedan ofrecer mejores servicios o precios más competitivos.

La apuesta española por la calidad en las infraestructuras es una realidad. Según los datos del Ministerio de Fomento, España se sitúa entre los diez primeros países de la clasificación mundial en calidad de las infraestructuras portuarias y en la quinta posición de Europa, mientras que en conectividad marítima ocupa la decimoprimera posición a nivel mundial y mantiene el quinto puesto a nivel europeo ${ }^{28}$.

Los datos de los puertos españoles ponen de relieve la pujanza del sector, ya que en 2017 se produjo un nuevo récord de tráfico, alcanzándose los 545 millones de toneladas de mercancías; de esta forma se confirma una curva ascendente, puesto que es el sexto año consecutivo que se bate este récord de tráfico ${ }^{29}$.

implementado medidas para reducir el impacto ambiental del sector marítimo y las fortalezas y debilidades que presentan, TAUDAL POULSEN, R., PONTE, S. y SORNN-FRIESE, H., "Environmental upgrading in global value chains: The potential and limitations of ports in the greening of maritime transport”, Geoforum, vol. 89, febrero 2018, pp. 83-95.

${ }^{28} \mathrm{El} 60 \%$ de las exportaciones y el $75 \%$ de las importaciones españolas tienen lugar a través de los puertos. El sector portuario genera 275.000 puestos de trabajo directos, indirectos e inducidos, y aporta más del $2 \%$ de nuestro PIB.

${ }^{29}$ En el año 2016 se alcanzaron los 509 millones de toneladas, incrementándose un 17\% los pasajeros y un $10,5 \%$ las mercancías. Según los datos ofrecidos por Puertos del Estado, el de Algeciras fue el primer puerto español en superar los 100 millones de toneladas, situándose así el primero del Mediterráneo y entre el cuarto y el quinto de Europa. PUERTOS DEL ESTADO (MINISTERIO DE FOMENTO), Plan de inversiones de accesibilidad portuaria 2017-2021. Inversiones portuarias 2017, 9 de enero de 2017, disponible en <http://www.puertos.es/es-es/Documents/PresentacionMinistroPuertosEnero2017.pdf> 
El texto refundido de la Ley de Puertos del Estado y de la Marina Mercante incluye en su articulado una serie de distinciones entre los puertos en función de las características de su tráfico y de la competencia administrativa ${ }^{30}$. Si bien la descarbonización del sector marítimo afecta a cualquier ámbito de la actividad portuaria, en este estudio nos vamos a centrar en los puertos de interés general, puesto que en ellos se efectúan actividades comerciales marítimas internacionales y el volumen anual de su actividad presenta unos niveles relevantes.

La perspectiva ambiental en el sistema portuario de titularidad estatal se regula en el título IV del texto refundido, que comprende las previsiones para la prevención y lucha contra la contaminación en el dominio público portuario, la recepción de desechos y residuos procedentes de buques, las obras de dragado y los planes de emergencia y seguridad $^{31}$.

\section{Calidad del aire en el ámbito portuario}

Siendo el objeto de nuestro estudio la descarbonización en el sector portuario, cabe referirse al contexto normativo sobre la calidad del aire. La norma comunitaria de referencia es la Directiva 2008/50/CE, relativa a la calidad del aire ambiente y una atmósfera más limpia en Europa ${ }^{32}$. En esta disposición se definen y establecen los objetivos de calidad del aire para evitar, prevenir o reducir los efectos nocivos para la salud humana y el medio ambiente en su conjunto. Asimismo, la norma comunitaria establece medidas para evaluar la calidad del aire ambiente basándose en métodos y criterios comunes ${ }^{33}$.

\footnotetext{
${ }^{30}$ Real Decreto Legislativo 2/2011, de 5 de septiembre, por el que se aprueba el Texto Refundido de la Ley de Puertos del Estado y de la Marina Mercante (BOE, núm. 253, de 20 de octubre de 2011).

${ }^{31} \mathrm{El}$ artículo 62 prohíbe las emisiones contaminantes en el dominio público portuario procedentes de buques o de medios flotantes de cualquier tipo.

${ }^{32}$ Directiva 2008/50/CE del Parlamento Europeo y del Consejo, de 21 de mayo de 2008, relativa a la calidad del aire ambiente y a una atmósfera más limpia en Europa (DO L 152, de 11 de junio de 2008). También cabe mencionar la Directiva 2016/802/UE del Parlamento Europeo y del Consejo, de 11 de mayo de 2016, relativa a la reducción del contenido de azufre de determinados combustibles líquidos (DO L 132, de 21 de mayo de 2016). Mediante esta disposición se fija el contenido máximo autorizado de azufre del fuelóleo pesado, del gasóleo, del gasóleo para uso marítimo y del combustible diésel para uso marítimo autorizados en la Unión.

${ }^{33}$ En relación con el marco normativo sobre la calidad del aire, FACCIOLI, C., La protección de la calidad del aire: Especial referencia a la actuación local, Publicacions URV, Tarragona, 2018.
} 
La Directiva hace referencia a la importancia de combatir las emisiones de contaminantes en la fuente, lo que es de especial interés para el sector portuario por la necesidad de adoptar medidas para la reducción de las emisiones en cualquiera de sus actividades. La norma comunitaria insta a la adopción de medidas de reducción de emisiones más eficaces a todos los niveles, local, nacional y comunitario. A este respecto, debemos recordar las obligaciones de las autoridades portuarias en el impulso de estas medidas; como se analizará con posterioridad, son numerosos los puertos en los que se han adoptado planes específicos para la mejora de la calidad del aire.

La reducción de emisiones y la mejora de la calidad del aire exigen un conocimiento previo sobre los datos reales de emisiones y su nivel de contaminación. También desde los puertos se puede llevar a cabo una interesante labor de recopilación de información que permita conocer de primera mano cuál es su contribución a las emisiones totales generadas en su entorno. Estas cifras adquieren especial significación en zonas saturadas desde el punto de vista de la contaminación atmosférica, en las que se hace imprescindible adoptar medidas para su reducción y control.

En el ámbito comunitario también cabe hacer referencia a la Directiva 2016/2284/UE, relativa a la reducción de las emisiones nacionales de determinados contaminantes atmosféricos ${ }^{34}$. Esta disposición impone obligaciones más estrictas sobre la calidad del aire y establece nuevos techos nacionales de emisión para el dióxido de azufre, los óxidos de nitrógeno, los compuestos orgánicos volátiles no metánicos, el amoniaco y las partículas finas; además, dispone la elaboración, adopción y aplicación de programas nacionales de control de la contaminación atmosférica, y el seguimiento de las emisiones y sus efectos. Sin embargo, la norma comunitaria excluye expresamente de los compromisos nacionales de reducción de emisiones de los cinco contaminantes mencionados a las emisiones del tráfico marítimo internacionales y a las emisiones del tráfico marítimo nacional desde y hacia las islas Canarias, los departamentos franceses de ultramar, Madeira y Azores ${ }^{35}$.

\footnotetext{
${ }^{34}$ Directiva 2016/2284/UE del Parlamento Europeo y del Consejo, de 14 de diciembre de 2016, relativa a la reducción de las emisiones nacionales de determinados contaminantes atmosféricos, por la que se modifica la Directiva 2003/35/CE y se deroga la Directiva 2001/81/CE (DO L 344, de 17 de diciembre de 2016).

${ }^{35}$ Artículo 4 de la Directiva 2016/2284/UE.
} 
A nivel nacional, la norma de referencia es la Ley 34/2007, de 15 de noviembre, de calidad del aire y protección de la atmósfera, que, siguiendo las directrices comunitarias, tiene por fin evitar o, en su caso, reducir los daños que la contaminación atmosférica puede originar en las personas, el medio ambiente y demás bienes de cualquier naturaleza; para ello, establece las bases en materia de prevención, vigilancia y reducción de este tipo de contaminación. La Ley identifica un conjunto de actividades potencialmente contaminadoras de la atmósfera, que constituyen su ámbito de aplicación. En el sector específico del transporte marítimo, se hace referencia a las embarcaciones y al tráfico marítimos, comprendiendo el tráfico marítimo nacional, la flota pesquera nacional y el tráfico marítimo internacional (incluidos bunkers internacionales $)^{36}$.

En materia de evaluación y gestión de la calidad del aire, las Administraciones públicas están obligadas a adoptar las medidas necesarias para mantener y, en su caso, mejorar la calidad del aire, así como a cumplir los objetivos de niveles máximos admitidos para cada contaminante atmosférico en función de su incidencia sobre la salud y el medio ambiente.

Las comunidades autónomas y los entes locales son los responsables de evaluar la calidad del aire en su ámbito territorial y, en consecuencia, establecer una zonificación según los niveles de los contaminantes para los que se hayan establecido objetivos de calidad del aire. Esta diferenciación en zonas del territorio habrá de tenerse en cuenta por las Administraciones públicas en la elaboración y aprobación de planes urbanísticos y de ordenación del territorio, así como en la tramitación de los procedimientos de autorización de actividades e instalaciones potencialmente contaminadoras de la atmósfera. Por lo tanto, es determinante el ámbito territorial en el que se ubica un puerto y el nivel de contaminación atmosférica de la zona en la que se incluye, puesto que puede condicionar su desarrollo futuro; por ejemplo, en el caso de iniciativas de reconversión de sus actividades y servicios portuarios, que pueden verse limitados por los niveles de contaminación máximos alcanzados al encontrarnos ante actividades potencialmente contaminadoras de la atmósfera.

\footnotetext{
${ }^{36}$ Anexo IV de la Ley 34/2007, en el que se incluye el catálogo de actividades potencialmente contaminadoras de la atmósfera. Real Decreto 239/2013, de 5 de abril, por el que se establecen las normas para la aplicación del Reglamento (CE) n. ${ }^{\circ}$ 1221/2009 del Parlamento Europeo y del Consejo, de 25 de noviembre de 2009, relativo a la participación voluntaria de organizaciones en un sistema comunitario de gestión y auditoría medioambientales (EMAS) (BOE, núm. 89, de 13 de abril de 2013).
} 
Asimismo, debemos recordar que el ámbito portuario también estará condicionado por los instrumentos de planificación adoptados a nivel estatal, autonómico y local a fin de prevenir y reducir la contaminación atmosférica. Con especial atención para aquellas zonas que sufran saturación ante ciertos contaminantes y que exijan medidas específicas para reducir sus efectos en la calidad del aire. En estos casos, también se contará con la colaboración de representantes del sector portuario a la hora de concretar dichas medidas $^{37}$.

\section{Medidas de protección ambiental y calidad portuaria de carácter voluntario}

Entre las medidas de carácter voluntario, debemos mencionar el Sistema Comunitario de Gestión y Auditoría Medioambientales (EMAS), que permite la participación de organizaciones de dentro y fuera de la Unión y que busca crear un sistema único con credibilidad y evitar la proliferación de diferentes sistemas nacionales ${ }^{38}$. El objetivo del EMAS es la mejora continua del comportamiento medioambiental de una organización a través de una política ambiental de empresa basada en una evaluación ambiental sistemática, objetiva y periódica, y de un programa medioambiental estructurado en objetivos, plazos y medios.

Además de esta iniciativa comunitaria, destacamos la UNE-EN-ISO 14001, que comparte con el EMAS el fomento de la protección medioambiental y la prevención de la contaminación. La norma UNE-EN-ISO 14001 comprende un conjunto de criterios, aceptados internacionalmente, que han cumplir los sistemas de gestión medioambiental de las organizaciones para poder ser certificados y que aspiran a la mejora continua en materia medioambiental ${ }^{39}$.

\footnotetext{
${ }^{37}$ En materia de gestión y planificación de la calidad del aire, debemos remitirnos a las previsiones del Real Decreto 102/2011, de 28 de enero, relativo a la mejora de la calidad del aire (BOE, núm. 25, de 29 de enero de 2011).

${ }^{38}$ Reglamento (CE) n. ${ }^{\circ}$ 1221/2009 del Parlamento Europeo y del Consejo, de 25 de noviembre de 2009, relativo a la participación voluntaria de organizaciones en un sistema comunitario de gestión y auditoría medioambientales (EMAS) (DO L 342, de 22 de diciembre de 2009). Real Decreto 239/2013, de 5 de abril, por el que se establecen las normas para la aplicación del Reglamento (CE) n. ${ }^{\mathbf{1}}$ 1221/2009 del Parlamento Europeo y del Consejo, de 25 de noviembre de 2009, relativo a la participación voluntaria de organizaciones en un sistema comunitario de gestión y auditoría medioambientales (EMAS) (BOE, núm. 89, de 13 de abril de 2013).

${ }^{39}$ Sobre esta materia, DOPAZO FRAGUÍO, P., El régimen jurídico de las marcas de calidad ambiental: etiqueta ecológica y tutela ambiental, Exlibris, Madrid, 2001; DOPAZO FRAGUÍO, P., "Gestión medioambiental y etiquetado ecológico: sistemas jurídicos europeos para promover la calidad ambiental
} 
Un importante número de puertos españoles se han adherido al EMAS y actualizan periódicamente su participación mediante las declaraciones validadas por un verificador medioambiental acreditado ${ }^{40}$. Asimismo, desde el propio Ministerio de Fomento se elaboró en 2011 una "Guía de Buenas Prácticas" para que las actividades portuarias se sometieran a un sistema de gestión ambiental basado en la norma ISO.

Además de estas medidas, en el ámbito portuario destacan las experiencias de iniciativas y acuerdos específicos y voluntarios a los que se han adherido autoridades portuarias de todo el mundo. En este sentido, podemos citar la World Ports Climate Declaration, adoptada en 2008, por la que los puertos participantes, reconociendo la influencia de las actividades portuarias en la emisión de gases de efecto invernadero, se comprometieron a la implementación de medidas que redujeran la presión ambiental de sus actividades.

Una de las iniciativas de mayor interés es la certificación ambiental PERS (Port Environmental Review System), otorgada por la Asociación Europea de Puertos tras un informe de auditoría realizado por EcoPorts ${ }^{41}$. Su principal característica es que es la única certificación de gestión ambiental que está diseñada específicamente para el sector portuario y que tiene origen en los propios puertos. La finalidad última de este instrumento es la consecución de una gestión ambiental eficaz que permita alcanzar los objetivos de desarrollo sostenible que son exigibles al sector. La implementación de estos objetivos se certifica de forma independiente por Lloyd's Register Quality Assurance, que otorga un certificado con una validez de dos años.

Los efectos que sobre la actividad portuaria tiene la consecución de esta certificación son evidentes, puesto que implica una mayor seguridad y garantía para los actores de la industria marítima. En definitiva, mejora su competitividad frente a otros puertos, ya que ofrece una distinción en el cumplimiento de unos estándares ambientales y de calidad que, cada día más, se valoran de forma positiva para la elección de un puerto por parte de sus potenciales clientes.

(EMAS y EEE)", Dopazo Fraguío, P., Derecho administrativo del medio ambiente. Temas y prácticas de actualidad jurídica, Universidad Complutense de Madrid, Servicio de Publicaciones, Madrid, 2018, pp. 81-110.

${ }^{40}$ A título ejemplificativo podemos citar el puerto de A Coruña, que incluye en su página web los datos correspondientes a su adhesión al EMAS y al ISO 14001. <http://www.puertocoruna.com/es/autoridadportuaria/sostenibilidad/medio-ambiente/declaracion.html $>$.

${ }^{41}$ Más información sobre esta iniciativa en <http://https://www.ecoports.com/pers>. 
Asimismo, también implica el cumplimiento de la normativa vigente ${ }^{42}$ y un compromiso real con el medio ambiente en cada uno de los procesos que se desarrollan en la actividad portuaria. Elementos que se valoran positivamente por parte de las instituciones comunitarias y que son determinantes para la obtención de fondos europeos que permitan la adopción e implementación de las últimas técnicas y mecanismos que favorezcan el mantenimiento y la mejora de estos niveles de protección.

\section{Combustibles alternativos: el impulso desde los puertos}

El fomento de combustibles alternativos, como el GNL, es una realidad que está incentivándose como una medida de control y reducción de los gases de efecto invernadero $^{43}$. La actuación de los puertos a este respecto es clave, puesto que, al ser nudos de conexión entre el transporte marítimo y otros medios complementarios y piezas clave de la cadena logística, pueden actuar como impulsores de estas iniciativas. No obstante, esta finalidad requiere una labor previa por parte de los puertos para dotarse de las infraestructuras y dotaciones de suministro necesarias.

A nivel europeo, cabe destacar la labor realizada en los puertos de Estocolmo y Hamburgo, que han sido pioneros en la implantación de medidas de eficiencia energética, combustibles alternativos y reducción de emisiones. En nuestro país ya nos encontramos con algunos ejemplos en este sentido, como es el caso del Port de Barcelona, que fomenta el empleo de este combustible en los buques, los camiones y la maquinaria portuaria.

Con la adopción en 2014 de la Directiva comunitaria que reguló la implantación de estructuras para los combustibles alternativos, se comenzó a gestar un esquema normativo que impulsaba esta nueva etapa en el sector marítimo ${ }^{44}$. La transposición de

\footnotetext{
${ }^{42}$ En ámbitos como el control y la reducción de los residuos generados o el nivel de emisiones, entre otros.

${ }^{43}$ En relación con las limitaciones de los combustibles alternativos en el transporte marítimo, GILBERT, P., WALSH, C., TRAUT, M., KESIEME, U., PAZOUKI, K., y MURPHY, A., “Assessment of full lifecycle air emissions of alternative shipping fuels", Journal of Cleaner Production, vol. 172, núm. 20, 2018, pp. 855-866.

${ }^{44}$ Directiva 2014/94/UE del Parlamento Europeo y del Consejo, de 22 de octubre de 2014, relativa a la implantación de una infraestructura para los combustibles alternativos (DO L 307, de 28 de octubre de 2014). BLANCO MONGE, S., “Observatory on 94/2014/EU Implementation. In relation to the LNG
} 
esta norma comunitaria en nuestro país dio como resultado la adopción del Real Decreto 639/2016, mediante el que se establece un marco de medidas para la implantación de una infraestructura para los combustibles alternativos ${ }^{45}$; la finalidad última de esta disposición, siguiendo las previsiones de la Directiva comunitaria, es reducir el impacto medioambiental del transporte y su dependencia respecto del petróleo y mitigar el impacto medioambiental del transporte.

El Marco de Acción Nacional adoptado por la Administración General del Estado fue aprobado a fines de $2016^{46}$ y realiza un profundo análisis sobre las distintas alternativas de combustibles, justificando la apuesta en el sector del transporte marítimo por el GNL y por la conexión a la red eléctrica de los buques durante su atraque, lo que les permite apagar sus motores auxiliares.

En este texto se realiza una mención específica al Proyecto CORE LNGas HIVE - Core Network Corridors and Liquefied Natural Gas, cuyo objetivo es fomentar este combustible en el transporte marítimo, poniendo el énfasis en la dotación de las infraestructuras portuarias que permitan dar respuesta a las necesidades de los buques que empleen GNL y establecer una cadena logística en consonancia con esta apuesta. De esta forma, y atendiendo al incremento en la demanda que se prevé para los próximos años, en el seno de este proyecto se están llevado a cabo estudios y experiencias piloto para extrapolar sus resultados a todo el territorio nacional.

Otra de las iniciativas más destacadas que se están desarrollando actualmente es el proyecto S/F SamueLNG for a Blue Atlantic $\mathrm{Arch}^{47}$, cofinanciado por la Unión Europea

market in the Maritime Transport", Green Energy Ports Conference, Vigo, 27-29 de junio de 2017, $<$ http://gepc.bluegrowthvigo.eu/>.

${ }^{45}$ Real Decreto 639/2016, de 9 de diciembre, por el que se establece un marco de medidas para la implantación de una infraestructura para los combustibles alternativos (BOE, núm. 298, de 10 de diciembre de 2016). Según lo dispuesto en el artículo 2 de esta disposición, los combustibles alternativos se definen en los siguientes términos: "[...] combustibles o fuentes de energía que sustituyen, al menos en parte, a los combustibles fósiles clásicos como fuente de energía en el transporte y que pueden contribuir a la descarbonización de estos últimos y a mejorar el comportamiento medioambiental del sector del transporte. Incluyen, entre otros: la electricidad, el hidrógeno, los biocarburantes, tal como se definen en el artículo 2, punto 2, del Real Decreto 1597/2011, de 4 de noviembre, por el que se regulan los criterios de sostenibilidad de los biocarburantes y biolíquidos, el Sistema Nacional de Verificación de la Sostenibilidad y el doble valor de algunos biocarburantes a efectos de su cómputo, los combustibles sintéticos y parafínicos, el gas natural, incluido el biometano, en forma gaseosa (gas natural comprimido (GNC)) y en forma licuada (gas natural licuado (GNL)), y el gas licuado del petróleo (GLP)".

${ }^{46}$ El Marco de Acción Nacional de Energías Alternativas en el Transporte fue aprobado en Consejo de Ministros celebrado el 9 de diciembre de 2016.

${ }^{47} \mathrm{La}$ denominación S/F SamueLNG proviene de las siglas de Spanish/French Sustainable Atlantic Motorways of the seas Using as fuel for Engine LNG. 
y en el que participan socios de España, Francia y Países Bajos, que aspira a fomentar las infraestructuras que permitan la implantación del GNL como combustible en el sector marítimo. El ámbito de actuación es el corredor atlántico, una de las principales rutas marítimas europeas. En el caso español, participan en el proyecto el Puerto de Gijón y el Puerto de Vigo, cuyos objetivos se centran en la adaptación de los buques para el empleo de este combustible y en la adaptación de la cadena logística a las nuevas necesidades de suministro.

\section{Puertos nacionales innovadores: el Port de Barcelona y el Puerto de Vigo}

a) El Port de Barcelona y su apuesta por la sostenibilidad económica, social y ambiental

Hemos seleccionado este puerto porque se organiza en tres unidades de negocio, lo que también nos permite abarcar un campo de análisis más amplio. Estas tres unidades son: el puerto comercial, dedicado a tráficos específicos (cruceros, contenedores, graneles, barcos de ferry y short sea, y tráficos de vehículos nuevos); el puerto logístico, vinculado al puerto comercial y elemento clave para el desarrollo del modelo "puerto en red"; y el puerto ciudad, destinado a los usos urbanos, náuticos y deportivos.

En el año 1998 el Port de Barcelona elaboró su I Plan Estratégico con el fin de desarrollar un objetivo común a todos los actores del sector portuario: convertir Barcelona en la primera plataforma logística euromediterránea. Desde entonces se han sucedido los instrumentos de planificación y en la actualidad se encuentra vigente el III Plan Estratégico 2015-2020 48 , que continúa compartiendo con el primer plan la misión que ha de guiar a este puerto: “[...] contribuir a la competitividad de los clientes del Port mediante la prestación de servicios eficientes que respondan a sus necesidades de transporte marítimo, transporte terrestre y servicios logísticos".

Los tres ejes temáticos en torno a los que se estructuran los objetivos estratégicos del Plan son el crecimiento, la competitividad y la sostenibilidad, donde crecimiento y competitividad van de la mano, por cuanto se hace hincapié en que el crecimiento no es un fin en sí mismo, sino que la finalidad del puerto es la creación de riqueza para su

\footnotetext{
48 El texto íntegro del Plan se puede consultar en el siguiente enlace: <http://content.portdebarcelona.cat/cntmng/guestDownload/direct/workspace/SpacesStore/fbb95a667f28-44cf-af32-000c7f113052/III_PLA_ESTRATEGIC_PBCN_es.pdf>
} 
área de influencia, lo que solo es posible ofreciendo elementos de competitividad a las empresas.

Por su parte, la sostenibilidad se dirige a tres ámbitos: sostenibilidad económica, social y ambiental. La sostenibilidad se desarrolla a través de cinco objetivos estratégicos que persiguen mejorar la accesibilidad y la movilidad, potenciar la intermodalidad, crecer de forma sostenible, mejorar la integración del Port y la ciudad, y convertir el puerto en uno de los principales polos de la industria náutica del Mediterráneo. El crecimiento sostenible desarrolla la triple perspectiva económica, social y ambiental; nos centraremos en las iniciativas ambientales, que siguen las líneas de actuación que ya hemos comentado en apartados anteriores.

Entre las prioridades ambientales se encuentran la reducción de las emisiones de gases de efecto invernadero mediante la implantación de políticas de ahorro energético y la promoción del uso de fuentes de energía alternativas. Como ya se ha analizado en apartados anteriores, el Port de Barcelona apuesta por el GNL tanto para los servicios portuarios marítimos como los terrestres, y por el fomento de la intermodalidad.

Entre las propuestas que consideramos más relevantes se encuentra la promoción de la fachada marítima mediterránea como punto de entrada en Europa de las mercancías provenientes de Asia o África, ofreciendo de esta forma una ruta más eficiente y sostenible frente a los puertos del norte al reducir los recorridos, las emisiones y los costes externos. Según los datos ofrecidos por el Port, los puertos mediterráneos ofrecen una ventaja competitiva medioambiental que se traduce en una reducción de cuatro o cinco días menos de navegación, lo que supone un ahorro medio estimado del $20 \%$ de emisiones de dióxido de carbono y, a la vez, reduce la congestión de las redes de transporte del norte de Europa y los costes logísticos ${ }^{49}$.

El Port de Barcelona también ha elaborado el Plan de Sostenibilidad Sectorial, que, partiendo de las directrices del Plan Estratégico, desarrolla una iniciativa configurada por cuatro etapas: proceso de adhesión de empresas y Administraciones de la comunidad logística portuaria, elaboración de la primera "Memoria de Sostenibilidad 
Sectorial", elaboración y ejecución del Plan de Acción, y, por último, revisión de las expectativas de los grupos de interés (cada dos o tres años).

El elemento clave del Plan son los datos suministrados por las empresas y Administraciones de la comunidad logística portuaria y aquellos de los que dispone la Autoridad Portuaria de Barcelona. Por lo tanto, es esta información suministrada la base sobre la que se identifican las amenazas y oportunidades, a partir de la cual se elabora un documento DAFO que permite definir los objetivos anuales y las iniciativas para su consecución. A principios de 2018, había 54 organizaciones de distintos ámbitos de la actividad portuaria que formaban parte de esta iniciativa; para integrarse en ella tan solo habían completado una encuesta sobre cuál era su actuación desde la perspectiva de la sostenibilidad.

En 2017 se elaboró la primera memoria, que comprendía, entre otros contenidos, la participación de los grupos de interés identificados y los indicadores de ética e integridad, y estudiaba de forma individualizada cada una de las dimensiones (económica, social y ambiental). En la dimensión ambiental se analizaban los consumos de energía y agua en el recinto portuario, las emisiones a la atmósfera, los efluentes y residuos, el transporte y la prevención de riesgos ambientales.

La concepción de la sostenibilidad ambiental como un elemento de competitividad frente a otros puertos europeos es una de las iniciativas desarrolladas por el Port de Barcelona. A este respecto, podemos destacar dos herramientas que proporcionan información sobre el impacto de la actividad portuaria. La primera de ellas sería la ecocalculadora, que mide las emisiones de $\mathrm{CO}_{2}$ que genera el transporte de un contenedor entre Europa y el resto del mundo a través del Port de Barcelona, a la vez que ofrece comparativas con otros puertos ${ }^{50}$.

Desde 2016 se ha implantado una herramienta más elaborada, Port Links, que muestra una cadena de transporte completa para un contenedor (integrando la oferta de servicios marítimos regulares, ferroviarios y en camión del puerto), representa la ruta en un mapa

${ }^{50} \mathrm{La}$ guía para el uso de esta herramienta y el acceso a esta se encuentran disponibles en <http://www.portdebarcelona.cat/es/web/port-dels-negocis/ecocalculadora>. 
y ofrece datos sobre los tiempos de tránsito, distancias, emisiones de dióxido de carbono y otros contaminantes, externalidades del transporte e información del paso portuario ${ }^{51}$.

Entre los instrumentos de planificación elaborados por el Port de Barcelona, debemos destacar el Plan de Mejora de la Calidad del Aire; este instrumento parte de la normativa autonómica y de los planes elaborados por la Generalitat y por el Ayuntamiento de Barcelona $^{52}$. El primer dato significativo, y que va a condicionar las medidas adoptadas, es que el Port de Barcelona está incluido en las zonas de protección especial del ambiente atmosférico en relación con el dióxido de nitrógeno y las partículas en suspensión con un diámetro inferior a las diez micras ${ }^{53}$. A fin de desarrollar las acciones concretas para restablecer la calidad del aire, la Generalitat adoptó el Plan de Actuación para la Mejora de la Calidad del Aire en las Zonas de Protección Atmosférica; según los datos de este plan, el ámbito del Port de Barcelona representa el 14\% de las emisiones de óxidos de nitrógeno y el 23\% de las partículas en suspensión de diámetro inferior a las 10 micras del total de emisiones en la zona de protección especial del ambiente atmosférico ${ }^{54}$.

En este contexto, el Port de Barcelona elaboró el Plan de Mejora de la Calidad del Aire, que comprende actuaciones para la reducción de las emisiones de buques, del tráfico, de la maquinaria de terminal, de las obras portuarias y de la manipulación de sólidos a granel, el impulso del transporte ferroviario y el short sea shipping, nuevos accesos, la movilidad sostenible y la red de vigilancia de la calidad del aire.

\section{b) El puerto de Vigo como referente para el crecimiento azul}

\footnotetext{
51 Para acceder a esta herramienta y conocer sus principales características, <http://www.portdebarcelona.cat/port-links>.

52 Plan de Mejora de la Calidad del Aire de Barcelona 2015-2018, adoptado por el Ayuntamiento de Barcelona.

${ }^{53}$ La norma de referencia a nivel autonómico es la Ley 22/1983, de 21 de noviembre, de protección del Ambiente Atmosférico (Diario Oficial de la Generalitat de Catalunya, núm. 385, de 30 de noviembre de 1983). Esta disposición tiene por objeto "establecer y regular los instrumentos y el procedimiento que se consideran necesarios para una actuación efectiva de las Administraciones públicas de Cataluña en el campo de la prevención, vigilancia y corrección de la contaminación atmosférica" (art. 1). Decreto 226/2006, de 23 de mayo, por el que se declaran zonas de protección especial del ambiente atmosférico diversos municipios de las comarcas de El Barcelonès, El Vallès Oriental, El Vallès Occidental y El Baix Llobregat para el contaminante dióxido de nitrógeno y para las partículas (Diario Oficial de la Generalitat de Catalunya, de 25 de mayo de 2006).
}

${ }^{54}$ Aprobado por el Acuerdo de Gobierno GOV/127/2014. 
En nuestro país cabe destacar los esfuerzos del Puerto de Vigo para implantar un sistema portuario sostenible. Siguiendo la línea de las certificaciones a las que hemos aludido con anterioridad, debemos mencionar que este puerto recibió en 2017 un informe de auditoría en el que se le reconocía como un puerto modelo en el cumplimiento de la política de protección ambiental y en lo que respecta a la consecución de los objetivos de desarrollo sostenible ${ }^{55}$.

El Puerto de Vigo hace varios años que está liderando una apuesta por la sostenibilidad en el sector portuario, lo que le ha llevado a desarrollar proyectos junto a otros puertos del mundo, europeos y norteamericanos, a fin de diseñar y concretar la definición del puerto para las próximas décadas. En definitiva, ha situado a Vigo en el mapa del sector portuario mundial, otorgándole una posición privilegiada y permitiendo un desarrollo paralelo de toda la actividad marítima de la ciudad, que se ve favorecida por esta apuesta por la sostenibilidad.

El Plan Blue Growth es la estrella de las iniciativas lideradas por la Autoridad Portuaria de Vigo y se ha proyectado como ejemplo a seguir por otras instituciones ${ }^{56}$. Esta iniciativa está implementando, desde una perspectiva integral, las propuestas de crecimiento azul planteadas desde las instancias comunitarias. A diferencia de otros proyectos realizados al amparo de esta línea política de la Unión Europea, lo novedoso de la iniciativa viguesa es que apuesta por un crecimiento azul que implique a todos los sectores, sin privilegiar a ninguno de ellos; un crecimiento azul real, y no parcelado, para todo el espacio marítimo-terrestre en el que se implementa este plan.

Los cuatro objetivos en torno a los cuales se estructuran los proyectos y las acciones integran las principales áreas de actuación en las que se vertebra el crecimiento azul ${ }^{57}$. En primer lugar, la innovación, marco básico de referencia para una estrategia que impulsa la eficiencia en la tecnología y que se apoya en la colaboración público-privada, vinculando la industria marítima con la investigación.

\footnotetext{
55 Según este informe, el Puerto de Vigo alcanza un elevado grado de cumplimiento respecto a los máximos certificados ambientales, logrando un 97,78\% de cumplimiento de las disposiciones previstas en el PERS, un 98,39\% según la norma ISO 14001 y un 98,44\% de conformidad con los criterios EMAS.

${ }^{56}$ Para más información sobre esta iniciativa, se puede consultar la web <http://bluegrowthvigo.eu/es\#>.

${ }^{57}$ BOTANA, C., "Blue Growth. An example on Integrated Maritime Policy. Potential transfer to other Ports!", Green Energy Ports Conference, Vigo, 27-29 de junio de 2017, $<$ http://gepc.bluegrowthvigo.eu/>.
} 
La perspectiva ambiental, en estrecha relación con la innovación, se marca como objetivo último que el Puerto de Vigo se convierta en el puerto verde de referencia del sur de Europa, para lo cual se fomentan las energías limpias, la implantación de procesos sostenibles en la actividad portuaria y la recuperación de espacios.

El tercero de los objetivos se refiere a la conectividad, vinculada a la transformación digital; comprende la digitalización de los procesos administrativos, el desarrollo de las herramientas TIC en la comunicación y la multiplicación de las líneas marítimas. Y el último de los objetivos se centra en las personas, desde la perspectiva del empleo a través de la formación y la integración de los sectores tradicionales hasta las propuestas centradas en la innovación social y el desarrollo de las humanidades, la historia del puerto y la historia marítima en general.

Debemos resaltar la capacidad de integración que presenta y, de forma muy destacada, la relevancia que el proyecto otorga a dos elementos clave para la perspectiva jurídica: por una parte, la apuesta por la innovación jurídica aplicada al sector marítimo y pesquero, y, por otra, la apuesta por un modelo de gestión administrativa que gira en torno a la participación activa y en el que la Autoridad Portuaria se erige en dinamizadora de este crecimiento azul.

Estas iniciativas se han proyectado hacia la Administración General del Estado, que también se ha adherido a este plan a través del Ministerio de Agricultura y Pesca, Alimentación y Medio Ambiente. En la misma línea, se ha firmado un protocolo de colaboración entre el citado Ministerio y el Puerto de Vigo para el impulso del crecimiento azul en el noroeste de España; su principal objetivo es la identificación de nuevas actividades de desarrollo económico y la promoción de la compatibilización de usos del espacio marítimo.

Desde la perspectiva de la descarbonización, el Puerto de Vigo se ha comprometido a reducir en un $30 \%$ sus emisiones y a alcanzar un $3 \%$ de autosuficiencia energética para el 2022. Entre las iniciativas propuestas para la consecución de estos objetivos destaca la implementación de la autosuficiencia con energía limpia en el Parque Nacional de las Islas Cíes, de forma que la demanda eléctrica y térmica generada por los miles de visitantes que recibe cada año este territorio pueda ser satisfecha y además se destine el 
excedente energético para implementar un sistema de movilidad sin emisiones y potabilizar el agua $^{58}$.

\section{El papel de las autoridades portuarias en el nuevo desarrollo de los puertos}

Las iniciativas analizadas ponen de manifiesto que los puertos están actuando como canalizadores y ejecutores de iniciativas pioneras en el desarrollo de un nuevo paradigma para el sector marítimo. El puerto, como elemento de conexión más cercano al operador del tráfico marítimo y nudo clave para la relación entre el espacio costero y marino de un territorio, está tomando la iniciativa ante la inactividad de otros sectores administrativos.

Esta realidad se pone de manifiesto a nivel global, y las propias iniciativas voluntarias a las que algunos puertos se han sumado para incrementar los controles y la calidad en su actuación son una buena prueba de su compromiso. Ante la incapacidad de la OMI para adoptar medidas internacionales eficaces, rápidas y efectivas, las limitaciones de la Unión Europea y la pasividad de los Estados y otros niveles administrativos, son las autoridades portuarias las que están impulsando el cambio.

En nuestro ordenamiento jurídico las autoridades portuarias se definen como organismos públicos con personalidad jurídica y patrimonio propios y plena capacidad de obrar, sometidos al ordenamiento jurídico privado, salvo en el ejercicio de las funciones de poder público ${ }^{59}$. Es relevante destacar que las autoridades portuarias se rigen por el principio general de autonomía funcional y de gestión, siempre en el respeto

\footnotetext{
${ }^{58}$ Esta iniciativa es la base del Proyecto TUTATIS-nZE Cíes (near Zero Emissions Cíes). En la memoria del Plan Blue Growth se definen las tecnologías que se van a implementar para dotar a las islas de autosuficiencia energética, que pasan por "la instalación de placas fotovoltaicas, la creación de una planta mareomotriz o undimotriz, la utilización de pilas de combustibles ubicadas en puntos de demanda de energía térmica y grupos diésel actualizados o reconvertidos a gas para un funcionamiento de emergencia. Todo esto se soportará con una Smart Grid, una infraestructura de almacenamiento de energía como baterías o un sistema para el uso de excedente eléctrico en la potabilización de agua". AUTORIDAD PORTUARIA DE VIGO, SECRETARÍA TÉCNICA INOVALABS, Blue Growth. Puerto de Vigo 20162020, Vigo, julio de 2016, p. 137.

${ }^{59}$ Sobre la naturaleza jurídica de las autoridades portuarias, DE ROJAS MARTÍNEZ-PARETS, F., El régimen de los puertos del Estado, Aranzadi, Cizur Menor (Navarra), 2011, p. 33 y ss.; MORALEDA SACEDA, M., "Organización portuaria del Estado", De Fuentes Bardají, J., Manual de dominio público marítimo-terrestre y puertos del Estado, Abogacía General del Estado. Dirección del Servicio Jurídico del Estado, Aranzadi, Cizur Menor (Navarra), 2011, p. 424 y ss.
} 
de las competencias atribuidas a Puertos del Estado (Ministerio de Fomento) y a las comunidades autónomas ${ }^{60}$.

Las autoridades portuarias son competentes para la prestación de los servicios generales, la gestión y el control de los servicios portuarios, incluyendo la coordinación del transporte en el ámbito portuario y del tráfico portuario (marítimo y terrestre) y el fomento de las actividades industriales y comerciales relacionadas. También ejercen sus competencias sobre la gestión del dominio público portuario, la ordenación de la zona de servicio del puerto y de los usos portuarios, y las obras y servicios del puerto.

La gestión económica que llevan a cabo estos organismos se rige por la premisa de la optimización y rentabilización del patrimonio y de los servicios asignados ${ }^{61}$. El régimen financiero de los puertos ha sido el elemento clave que ha condicionado la organización administrativa portuaria y, por tanto, se encuentra en el origen y en la futura evolución de las autoridades portuarias ${ }^{62}$. El texto refundido de la Ley de Puertos del Estado y de la Marina Mercante atribuye a estos organismos la gestión y administración de una serie de recursos, entre los que se encuentran los productos y las rentas de su patrimonio, las tasas portuarias, los ingresos de derecho privado obtenidos en el ejercicios de sus funciones, y las ayudas y subvenciones, entre otros. Estos recursos son gestionados y administrados por las autoridades portuarias sobre el principio de autonomía de gestión y en respeto a los principios de eficacia, eficiencia y sostenibilidad ambiental.

A este respecto, también cabe recordar que el régimen económico de los puertos de titularidad estatal se rige por el principio de autosuficiencia económica del sistema portuario en su conjunto y de cada una de las autoridades portuarias ${ }^{63}$. En el último Informe de Gestión del Sistema Portuario de Titularidad Estatal, elaborado por Puertos del Estado y correspondiente al año 2015, se pone de manifiesto que el principal

\footnotetext{
${ }^{60}$ La regulación de las autoridades portuarias se desarrolla en los artículos 24 a 34 (sección 2. a del título II) del Real Decreto Legislativo 2/2011, de 5 de septiembre, por el que se aprueba el Texto Refundido de la Ley de Puertos del Estado y de la Marina Mercante (BOE, núm. 253, de 20 de octubre de 2011).

${ }^{61}$ MARTÍNEZ MOYA, J. y FEO VALERO, M., "El papel de las autoridades portuarias en la elección del puerto: principales resultados", Petit Lavall, M. y Puetz, A., La eficiencia del transporte como objetivo de la actuación de los poderes públicos: liberalización y responsabilidad, Marcial Pons, Madrid, 2015, pp. 775-790.

${ }^{62}$ En relación con el régimen financiero de los puertos y su influencia en la organización administrativa y en los servicios portuarios, TRIAS PRATS, B., El régimen económico de los servicios portuarios en los puertos estatales, Iustel, Madrid, 2011, p. 131 y ss.
}

${ }^{63}$ Artículo 156 Real Decreto Legislativo 2/2011. 
volumen de negocios de las autoridades portuarias españolas lo constituyen las tasas de utilización, destacando también los ingresos procedentes de la ocupación del dominio público portuario; por lo tanto, estos constituirían los principales ingresos de los citados $\operatorname{organismos}^{64}$.

Hay que poner de manifiesto que la posición que ocupan es privilegiada, puesto que les permite actuar como puente entre el sector público y el privado, aglutinar y dar respuesta a las demandas de la industria marítima, adoptar medidas reguladoras que les permitan desarrollar una política de incentivos, y conectar su desarrollo con la sociedad $\mathrm{y}$, de forma más específica, con el modelo de ciudad ${ }^{65}$.

El primer elemento sería el social, la dependencia entre el puerto y la localidad en la que se ubica. Las ciudades con puerto se encuentran marcadas por su relación con este espacio; todas las localidades portuarias deben su idiosincrasia al impacto que genera

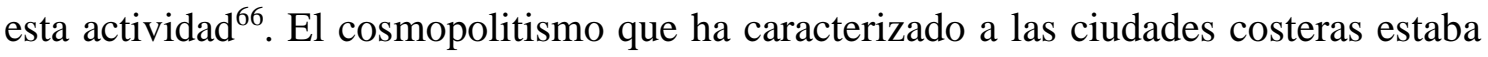
marcado por el trasiego de pasajeros y mercancías que generaban sus puertos, por lo que la historia de estos territorios no se entendería sin dichos espacios. En la actualidad,

\footnotetext{
${ }^{64} \mathrm{La}$ cifra de negocios del sistema portuario español en 2015 alcanzó los 1.045 millones de euros; las autoridades portuarias que lideraron estas aportaciones fueron: Barcelona (155,6 millones de euros), Valencia (127,3), Bahía de Algeciras (80), Baleares $(68,4)$, Las Palmas $(67,6)$ y Bilbao $(65,2)$. Las tasas de utilización ascendieron a 562,1 millones de euros, representando el 53,8\% de la cifra de negocios total. PUERTOS DEL ESTADO (MINISTERIO DE FOMENTO), Informe de Gestión del Sistema Portuario de Titularidad Estatal, 2015, <http://www.puertos.es/eses/Documents/INFORME\%20GESTION\%20SISTEMA\%20PORTUARIO\%202015.pdf>. Sobre el patrimonio de las autoridades portuarias, ACERO IGLESIAS, P., Organización y régimen jurídico de los puertos estatales, Aranzadi, Cizur Menor (Navarra), 2002, p. 131 y ss.
}

${ }^{65} \mathrm{~A}$ este respecto cabe recordar que los intereses públicos son la única guía para estos organismos. Como señala EGUINOA DE SAN ROMÁN, R., La gestión de los puertos de interés general, Atelier Libros, Barcelona, 2012, p. 116: "Las Autoridades Portuarias tienen encomendada la gestión de los puertos, no la gestión de intereses de los sectores portuarios relevantes; no hay una comunidad con una solidaridad de intereses para cuya gestión se crea la Autoridad Portuaria, por el encaje que pueden tener esos intereses con el interés público. Tampoco cabe distinguir entre intereses públicos e intereses 'privados' de las Autoridades Portuarias, entendidos éstos en el sentido de intereses de los 'asociados' que las integrarían en el supuesto de ser entes corporativos. El régimen jurídico dual (privado y público) aplicable a la actividad de las Autoridades Portuarias no obedece a que éstas persigan por una parte intereses de naturaleza privada y, por otro, intereses públicos: los intereses de estas entidades son enteramente públicos, con independencia del régimen jurídico de la actividad que han de desarrollar para conseguir su realización".

${ }^{66}$ Sobre la relevancia del concepto de ciudad y el análisis de cuatro de sus principales elementos definitorios (espacios públicos urbanos, infraestructuras y servicios urbanos, regulación del uso del suelo urbano y gobierno), AUBY, J., "La ciudad, ¿nueva frontera del Derecho Administrativo?”, TransJus Working Papers publications, núm. 3, 2018. En relación con la labor de las ciudades en materia de cambio climático, RUIZ DE APODACA, A., "Ciudades y cambio climático: Mitigación y adaptación", El cambio climático: Una mirada desde Cataluña. Primer Congreso Catalán de Derecho Ambiental, Tarragona, 17-19 de enero de 2018, <http://wwwa.fundacio.urv.cat/congressos/congres-catala-dretambiental/presentaciones>. 
pero ya desde otra perspectiva, el puerto marca el devenir de la ciudad y genera empleo y actividad económica, y las ciudades que son ajenas a esta realidad quedan desconectadas. La pesca, los contenedores de mercancías, la llegada de cruceristas, las regatas y un sinfín de ejemplos más marcan el día a día de la ciudad portuaria. Las autoridades portuarias están llamadas al fomento de esta relación entre el puerto y la ciudad, integrando a las comunidades locales y haciéndolas partícipes del desarrollo portuario $^{67}$.

El segundo elemento sería el económico, la creciente competitividad a nivel portuario también es uno de los elementos clave para analizar este liderazgo de la innovación desde las autoridades portuarias. En un contexto de desarrollo exponencial del transporte marítimo e incluso de apertura de nuevas rutas, la especialización y la innovación en el ámbito de las infraestructuras permiten a los puertos situarse en el mercado, ofreciendo a sus clientes un producto completo: calidad en el servicio y respeto máximo de los estándares ambientales.

Las autoridades portuarias adoptan una posición clave en materia de planificación, control y gestión energética en el puerto ${ }^{68}$. Y es desde esta posición como pueden influir en la descarbonización del transporte marítimo. En primer lugar, concienciando al sector

\footnotetext{
${ }^{67}$ Como se afirma en el Código de buenas prácticas para la integración social de los puertos, p. 10: "La erosión del respaldo social a los puertos marítimos ha sido reconocida sólo recientemente como una cuestión que requiere la atención de la gestión portuaria. Abordar la convivencia y el diálogo con las ciudades y las comunidades locales es todavía para muchas Autoridades Portuarias un proceso de aprendizaje difícil, ya que a menudo se suele ver como una distracción de la tradicional cultura de ingeniería y lógica comercial que rige las operaciones portuarias. La integración social es, sin embargo, una tarea perfecta para las Autoridades Portuarias, independientemente de cuál sea su estructura de propiedad u organizativa. Desde una perspectiva de gobernanza portuaria, está vinculada a la función de 'administrador de la comunidad' de la Autoridad Portuaria, función ésta que complementa las funciones tradicionales de propietario, regulador y operador. Esta función está intrínsecamente ligada a la naturaleza cambiante de las comunidades portuarias y las partes interesadas y tiene a la vez una dimensión económica y otra social". EUROPEAN SEA PORTS ORGANISATION, Código de buenas prácticas para la integración social de los puertos, Fundación Valencia Port, 2010. Sobre las medidas adoptadas para reducir el impacto ambiental de la actividad portuaria en su zona territorial de influencia, GONZÁLEZ AREGALL, M., BERGQVIST, R. y MONIOS, J., "A global review of the hiterland dimension of green port strategies", Transportation Research Part D, vol. 59, 2018, pp. 23-34.

${ }^{68}$ Como señalan Acciaro, Ghiara y Cusano, tras el estudio realizado en los puertos de Génova y Hamburgo: "[...] a suitable definition of the port authority as a promoter of energy management refers then to its role in planning, regulating and monitoring energy use within the port. In this function the port authority aims to improve energy efficiency by evaluating energyuse and implementing new policies and changes where necessary. The port authority promotes energy management by coordinating all aspects of energy management, from energy efficiency and reduction of the carbon footprint of the port to waste management and sustainable development by: -Encouraging the use of renewable/sustainable energy resources within an organisation or community, -Deriving solutions for carbon management, -Raising the profile of energy conservation". ACCIARO, M., GHIARA, H. y CUSANO, M., "Energy management in seaports: A new rol for port authorities", Energy Policy, núm. 71, 2014, p. 10.
} 
privado sobre su responsabilidad y ofreciendo datos reales de las emisiones que se producen. Para continuar con una política de incentivos que beneficien a aquellas compañías y empresas que desarrollen políticas activas para reducir su contribución al cambio climático. Todo ello, marcado por una labor previa de impulso y concienciación desde las Administraciones públicas para la creación de una infraestructura que permita el desarrollo de los combustibles alternativos para buques y la inversión para reducir las emisiones generadas por todos los actores implicados en los servicios portuarios ${ }^{69}$.

Esta perspectiva vinculada al sector privado se completa con la proyección de la influencia portuaria sobre el sector público. Cabe señalar que en gran medida son las autoridades portuarias las que están generando la política sobre el sector; están liderando proyectos, como es el caso del Puerto de Vigo, al que se suman la Administración autonómica y la estatal.

Las autoridades portuarias deben guardar este equilibrio entre su posicionamiento en el mercado y su vinculación social y ambiental. De hecho, el diálogo constante entre la ciudad y el puerto ha de ser una realidad que marque la toma de decisiones conjuntas en aquellos ámbitos de conexión ${ }^{70}$. A este respecto, podemos citar el propio articulado del texto refundido de la Ley de Puertos del Estado y de la Marina Mercante, donde se establece la obligación para las autoridades portuarias de elaborar anualmente un proyecto de plan de empresa en el que, además de recoger las previsiones económicofinancieras y los objetivos de gestión, se incluya una referencia obligatoria a los objetivos e indicadores de sostenibilidad ambiental del puerto; asimismo, la norma impone la obligatoriedad de acompañar el proyecto de plan de empresa con una memoria de sostenibilidad ${ }^{71}$.

\footnotetext{
69 A este respecto, cabe citar a Parejo Navajas cuando afirma que el cambio climático, más que un "problema global", es un "problema de múltiples niveles"; las autoridades portuarias actuarían como uno más de los niveles implicados en la gobernanza del cambio climático. PAREJO NAVAJAS, T., "Gobernar el cambio climático", Parejo Alfonso, L. (dir.), El derecho ante la innovación y los riesgos derivados del cambio climático, Tirant lo Blanch, Valencia, 2015, p. 57.

${ }^{70}$ A este respecto, podemos destacar que la presión de la ciudadanía ha sido clave para la adopción de determinadas políticas portuarias, como es el caso de los puertos de Los Ángeles y Long Beach, que adoptaron el Clean Air Action Plan como respuesta a las demandas sociales. Sobre esta iniciativa, GIULIANO, G. y LINDER, A., "Motivations for self-regulation: the clean air action plan", Energy Policy, vol. 59, 2013, pp. 513-522.

${ }^{71}$ Artículo 55 del texto refundido de la Ley de Puertos del Estado y de la Marina Mercante. Respecto a este precepto, González Laxe destaca que la normativa española sobre esta materia es una de las más avanzadas de Europa, puesto que no existe ninguna otra regulación sobre puertos que incluya indicadores
} 
En este contexto vamos a analizar las iniciativas del Port de Barcelona, líder en rentabilidad económica a nivel nacional ${ }^{72}$, que puede servir de ejemplo sobre cómo las autoridades portuarias nacionales están enfocando esta nueva etapa.

En el III Plan Estratégico 2015-2020 se define la misión que guía la labor de la autoridad portuaria de este organismo: "[...] liderar el desarrollo del Port de Barcelona, generar y gestionar infraestructuras y garantizar la fiabilidad de los servicios para contribuir a la competitividad de los clientes y crear valor para la sociedad". La visión de esta autoridad portuaria, compartida por el Port de Barcelona y que podemos definir como su lema, sería “Barcelona: puerto solución de Europa en el Mediterráneo".

En el Plan se identifican cuatro factores clave de éxito interno para la autoridad portuaria: la orientación al mercado, el liderazgo de la comunidad logística, la excelencia en la gestión y la coordinación interna, y el cumplimiento del marco legal y la autonomía de gestión. El Plan del Port de Barcelona identifica tres objetivos estratégicos específicos que han de ser alcanzados por la autoridad portuaria y que tienen por finalidad mejorar la eficiencia y orientación al cliente de sus procesos internos, adaptar sus recursos a los objetivos del Port y mejorar la capacidad de generar recursos.

Hay varios aspectos de estos factores que consideramos de especial interés, en especial, la correcta gestión del liderazgo. Este tema nos parece de relevancia por cuanto el estudio realizado pone de manifiesto cómo las autoridades portuarias están erigiéndose en promotoras de las políticas portuarias más innovadoras. Por tanto, y a la vista de la experiencia del Port de Barcelona, es importante conocer cuáles son las dificultades para gestionar este liderazgo; en el Plan Estratégico se pone de manifiesto que la prestación de los servicios portuarios implica la intervención de varias Administraciones y empresas privadas, si bien es la autoridad portuaria la responsable de ejercer la

de sostenibilidad. GONZÁLEZ LAXE, F., MARTÍN BERMÚDEZ, F., MARTÍN PALMERO, F. y NOVO-CORTI, I., "Sustainability and the Spanish port system. Analysis of the relationship between economic and environmental indicators", Marine Pollution Bulletin, núm. 113, 2016, p. 238.

${ }^{72} \mathrm{La}$ clave del éxito del Port de Barcelona toma como referencia aquellos elementos de su oferta que atraen a los clientes; según el III Plan Estratégico, son los siguientes: “[...] rutas más cortas hacia Europa para los mercados asiáticos y africanos, alternativa a los puertos de la fachada norte en la distribución en el Mediterráneo Occidental, integración en el principal nodo industrial y logístico del Mediterráneo, hinterland económicamente potente, red de terminales marítimas interiores, infraestructuras adecuadas y adaptadas a las necesidades futuras, buena capacidad y explotación portuaria con una cuidadosa planificación del territorio, buenas conexiones en el hinterland, productividad de las terminales, compromiso con la intermodalidad, y compromiso con la sostenibilidad y el medio ambiente". PORT DE BARCELONA, III Plan Estratégico 2015-2020, Port de Barcelona, p. 38. 
coordinación de los servicios y liderar los proyectos y actuaciones que permitan mejorar la competitividad. Por ello, se incide sobre la necesidad de disponer de estructuras adecuadas para gestionar este liderazgo y en el reconocimiento de esta posición por parte de todos los actores que integran el sector público y el privado.

Otro de los aspectos sobre los que se incide en este plan es la autonomía de gestión, señalándose que las limitaciones que el marco normativo vigente establece en cuanto a las funciones y competencias de las autoridades portuarias suponen un obstáculo para su crecimiento. Según esta propuesta, dotar a estos organismos de una mayor autonomía de gestión y de facilidades para el desarrollo de herramientas comerciales les permitiría competir en igualdad de condiciones con otros puertos europeos que disfrutan ya de estos instrumentos.

\section{CONCLUSIONES}

Frente a las aproximaciones tradicionales a los modelos de estudio del cambio climático y las alternativas energéticas, cabe analizar la perspectiva azul como una vía real sobre la que aún se debe reflexionar para ofrecer un marco de actuación coherente y respetuoso con las demandas socioeconómicas y la indispensable protección ambiental.

El transporte marítimo y el sector portuario actúan como modelos de esa dimensión, a la vez global y local, que impregna las medidas para la reducción de emisiones y la consecución de un modelo más sostenible de movilidad en el ámbito marítimo. Las iniciativas de cooperación internacional, de coordinación entre Estados y de proyectos conjuntos siguen siendo insuficientes para suplir la necesidad de adoptar una estrategia común a nivel global. En este contexto, marcado por las presiones de todos los sectores implicados, la limitación de la OMI es patente, y los plazos se prolongan. Sin embargo, y a pesar de estas deficiencias, en un modelo internacional de transporte es indispensable la adopción de directrices globales, por lo que deben continuar los proyectos de cooperación y debe fomentarse la adopción de acuerdos, estrategias, directrices o cualquier otra medida que persiga un marco común para todos los Estados.

Como complemento a esta labor, han de desarrollarse las iniciativas regionales, nacionales y locales. Repensar el modelo de gestión desde las Administraciones públicas es una de las prioridades que han de ser asumidas para implantar una política 
marítima que responda a las demandas medioambientales. La evolución desde una Administración pasiva hacia una Administración que promueva e impulse el crecimiento azul supone un cambio en la mentalidad de estos gestores administrativos.

El sector público también debe dar una respuesta a la nueva etapa en la que nos encontramos, en la que se está exigiendo a los actores privados de la industria marítima que asuman riesgos y se impliquen en la protección del espacio marítimo-terrestre y evolucionen con la implantación de nuevas tecnologías. Por lo tanto, no es suficiente con regular y aplicar; se debe avanzar en el cumplimiento de los principios básicos de la actuación administrativa, implicando a la sociedad en el crecimiento azul, haciendo participar a todos los sectores de la industria y a la ciudadanía.

El crecimiento azul supone la mejora y el avance de las localidades costeras y del entorno marítimo y marino. Es necesario integrar, y esta labor ha de estar liderada por aquellas Administraciones públicas que ostentan algún tipo de competencia sobre este ámbito, con el compromiso de actuar de forma coordinada para la adopción de medidas reales y efectivas. La actuación administrativa debe superar su imagen de obstaculizadora para transformarse en impulsora; no ha de definirse por las trabas administrativas, sino por las soluciones y los planes para la mejora.

En este contexto, las autoridades portuarias ocupan una posición preferente y están llamadas a implicarse en los cambios que se exigen al sector público. Los modelos que se están desarrollando en algunos puertos nacionales e internacionales nos ofrecen nuevas guías para adaptar la labor de estos organismos a las demandas de la industria y de la sociedad.

La renovación es una premisa que se ha de asumir como una constante para la transformación de un modelo de transporte que contribuye sobremanera al cambio climático. Esta renovación ha de estar guiada por la Administración y es en los puertos, núcleo de conexión entre el espacio terrestre y el marítimo, donde también es más factible que se produzca la conexión entre las medidas adoptadas desde la Administración y el sector marítimo. En una industria con un claro componente global y que está marcada por las directrices de la OMI y las iniciativas comunitarias, el impulso real puede venir de la mano del sector portuario.

Es en este contexto donde el sector público, a través de las autoridades portuarias pero sin olvidar su conexión con las restantes Administraciones públicas implicadas, ha de 
ser capaz de liderar la descarbonización, implicando a todos los actores privados y a la ciudadanía, abriendo una etapa marcada por la implantación de combustibles alternativos y de instrumentos para el fomento de la eficiencia energética.

En este contexto, las actuaciones locales pueden condicionar el desarrollo de un transporte marítimo con reducción de emisiones y un crecimiento realmente azul y no teñido de negro. La implantación de infraestructuras portuarias que fomentan los combustibles alternativos y el establecimiento de estándares de calidad y seguimiento de emisiones más estrictos que los previstos en la normativa vigente muestran una tendencia imparable y un modelo de mercado en los servicios portuarios que ofrece la reducción de las emisiones como un reclamo para sus potenciales clientes.

Las iniciativas adoptadas en los puertos de Barcelona y Vigo son claros ejemplos de este liderazgo desde las autoridades portuarias y de la capacidad de aglutinar esfuerzos privados y públicos. Hemos de destacar la proyección más allá de la vertiente puramente comercial y la necesidad de integrar el puerto en la ciudad y situarlo como el eje en torno al que giren las políticas de desarrollo socioeconómico y ambiental de la localidad.

\section{BIBLIOGRAFÍA}

ACERO IGLESIAS, P., Organización y régimen jurídico de los puertos estatales, Aranzadi, Cizur Menor (Navarra), 2002.

ACCIARO, M., GHIARA, H. y CUSANO, M., "Energy management in seaports: A new rol for port authorities”, Energy Policy, núm. 71, 2014.

AUBY, J., "La ciudad, ¿nueva frontera del Derecho Administrativo?”, TransJus Working Papers publications, núm. 3, 2018.

AUTORIDAD PORTUARIA DE VIGO, SECRETARÍA TÉCNICA INOVALABS, Blue Growth. Puerto de Vigo 2016-2020, Vigo, julio de 2016.

BARBERO, J. y RODRÍGUEZ TORNQUIST, R., "Transporte y cambio climático: hacia un desarrollo sostenible y de bajo carbono", Revista Transporte y Territorio, núm. 6, Universidad de Buenos Aires, 2012. 
BELLEVER CAPELLA, V., "El derecho al cambio climático: ascenso y decadencia del Protocolo de Kioto", Fernández Ruiz-Gálvez, E. (coord.), El futuro de los derechos humanos, Tirant lo Blanch, Valencia, 2016.

BLANCO MONGE, S., “Observatory on 94/2014/EU Implementation. In relation to the LNG market in the Maritime Transport", Green Energy Ports Conference, Vigo, 27-29 de junio de 2017, <http://gepc.bluegrowthvigo.eu/>.

BOTANA, C., "Blue Growth. An example on Integrated Maritime Policy. Potential transfer to other Ports!", Green Energy Ports Conference, Vigo, 27-29 de junio de 2017, $<$ http://gepc.bluegrowthvigo.eu/>.

CAMPINS ERITJA, M., "Entre la urgència a Fiji i la complexitat a Bonn. El lent camí per a la posada en marxa de l'Acord de París", Revista Catalana de Dret Ambiental, vol. VIII, núm. 2, 2017.

DE ROJAS MARTÍNEZ-PARETS, F., El régimen de los puertos del Estado, Aranzadi, Cizur Menor (Navarra), 2011.

DOERR, O., Políticas portuarias, Naciones Unidas, Santiago de Chile, 2011.

DOPAZO FRAGUÍO, P., El régimen jurídico de las marcas de calidad ambiental: etiqueta ecológica y tutela ambiental, Exlibris, Madrid, 2001.

DOPAZO FRAGUÍO, P., "Gestión medioambiental y etiquetado ecológico: sistemas jurídicos europeos para promover la calidad ambiental (EMAS y EEE)", Dopazo Fraguío, P., Derecho administrativo del medio ambiente. Temas y prácticas de actualidad jurídica, Universidad Complutense de Madrid, Servicio de Publicaciones, Madrid, 2018.

EGUINOA DE SAN ROMÁN, R., La gestión de los puertos de interés general, Atelier Libros, Barcelona, 2012.

EGUINOA DE SAN ROMÁN, R., Derecho comunitario y puertos de interés general, Atelier Libros, Barcelona, 2017.

EUROPEAN SEA PORTS ORGANISATION, Código de buenas prácticas para la integración social de los puertos, Fundación Valencia Port, 2010.

FACCIOLI, C., La protección de la calidad del aire: Especial referencia a la actuación local, Publicacions URV, Tarragona, 2018. 
FUENTES GASÓ, R., “Avances en la protección y conservación del medio marino español. El nuevo marco para la ordenación del espacio marítimo", Revista Catalana de Dret Ambiental, vol. VIII, núm. 1, 2017.

GILBERT, P., WALSH, C., TRAUT, M., KESIEME, U., PAZOUKI, K. y MURPHY, A., "Assessment of full life-cycle air emissions of alternative shipping fuels", Journal of Cleaner Production, vol. 172, núm. 20, 2018.

GIULIANO, G. y LINDER, A., "Motivations for self-regulation: the clean air action plan”, Energy Policy, vol. 59, 2013.

GONZÁLEZ AREGALL, M., BERGQVIST, R. y MONIOS, J., “A global review of the hiterland dimension of green port strategies", Transportation Research Part D, vol. 59, 2018 .

GONZÁLEZ LAXE, F., MARTÍN BERMÚDEZ, F., MARTÍN PALMERO, F. y NOVO-CORTI, I., "Sustainability and the Spanish port system. Analysis of the relationship between economic and environmental indicators", Marine Pollution Bulletin, núm. 113, 2016.

HOFFMANN, J. (coord), Review of Maritime Transport 2017, Naciones Unidas, UNCTAD/RMT/2017, 2017 <http://unctad.org/en/PublicationsLibrary/rmt2017_en.pdf>.

INTERNATIONAL CHAMBER OF SHIPPING, 2017 Annual Review, ICS, 2018, <http://www.ics-shipping.org/docs/default-source/ICS-Annual-Review-2017/ics-annualreview-2017.pdf?sfvrsn=10>.

INTERNATIONAL MARITIME ORGANIZATION, Third IMO GHC Study 2014, 2015 .

LAGO CANDEIRA, A., "El avance del Protocolo de Montreal hacia la mitigación del cambio climático (Enmienda de Kigali)”, López Ramón, F., Observatorio de Políticas Ambientales 2017, CIEMAT, Madrid, 2017.

LOZANO CUTANDA B., "Real Decreto 363/2017, de 8 de abril: la ordenación de los distintos usos y de las actividades económicas en el espacio marítimo", Diario La Ley, núm. 8999, 2017. 
MARTÍNEZ, A., "Gas Natural: situación, objetivos y oportunidades. ENDESA”, Green Energy Ports Conference, Vigo, 27-29 de junio de 2017, $<$ http://gepc.bluegrowthvigo.eu/>.

MARTÍNEZ MOYA, J. y FEO VALERO, M., "El papel de las autoridades portuarias en la elección del puerto: principales resultados", Petit Lavall, M. y Puetz, A., La eficiencia del transporte como objetivo de la actuación de los poderes públicos: liberalización y responsabilidad, Marcial Pons, Madrid, 2015.

MARTÍNEZ PÉREZ, E., "Mercado de emisiones y transporte internacional: acciones unilaterales e integración de la navegación aérea y el transporte marítimo en el ETS”, Sanz Rubiales, I. (coord.), Cambio climático y Unión Europea. Presente y futuro del mercado europeo de emisiones, Tirant lo Blanch, Valencia, 2014.

MORALEDA SACEDA, M., “Organización portuaria del Estado”, De Fuentes Bardají, J., Manual de dominio público marítimo-terrestre y puertos del Estado, Abogacía General del Estado. Dirección del Servicio Jurídico del Estado, Aranzadi, Cizur Menor (Navarra), 2011.

OLMER, N., COMER, B., ROY, B., MAO, X. y RUTHERFORD, D., Greenhouse gas emissions from global shipping, 2013-2015, The International Council on Clean Transportation, octubre de 2017, <https://www.theicct.org/publications/GHGemissions-global-shipping-2013-2015>.

PAREJO NAVAJAS, T., "Gobernar el cambio climático”, Parejo Alfonso, L. (dir.), El derecho ante la innovación y los riesgos derivados del cambio climático, Tirant lo Blanch, Valencia, 2015.

RODRÍGUEZ GARCÍA, N., "Responsabilidad del Estado y cambio climático: El caso Urgenda contra Países Bajos", Revista Catalana de Dret Ambiental, vol. VII, núm. 2, 2016, pp. 1-38.

RUIZ DE APODACA, A., "Ciudades y cambio climático: Mitigación y adaptación”, $E l$ cambio climático: Una mirada desde Cataluña. Primer Congreso Catalán de Derecho Ambiental, Tarragona, 17-19 de enero de 2018, $<$ http://wwwa.fundacio.urv.cat/congressos/congres-catala-dretambiental/presentaciones>. 
SANZ LARRUGA, J., "La nueva ordenación del espacio marítimo. Análisis del Real Decreto 363/2017, de 8 de abril”, Práctica urbanística. Revista mensual de urbanismo, núm. 150, 2018.

SARASÍBAR IRIARTE, M., "La Cumbre del Clima de París: ¿un avance importante en la política del cambio climático?”, Administración \& cidadanía: revista da Escola Galega de Administración Pública, vol. 11, núm. 2, 2016.

SARASÍBAR IRIARTE, M., "Cambio climático: su regulación jurídica como grave problema ambiental” López Ramón, F., Observatorio de Políticas Ambientales 2017, CIEMAT, Madrid, 2017.

SARASÍBAR IRIARTE, M., "Energías renovables y cambio climático: un binomio condenado a entenderse", Galán Vioque, R. y González Ríos, I. (dirs.), Derecho de las energías renovables y la eficiencia energética en el horizonte 2020, Aranzadi, Cizur Menor (Navarra), 2017.

SARASÍBAR IRIARTE, M., "Políticas y medidas de mitigación del cambio climático en el ámbito jurídico español”, El cambio climático: Una mirada desde Cataluña. Primer Congreso Catalán de Derecho Ambiental, Tarragona, 17-19 de enero de 2018, $<$ http://wwwa.fundacio.urv.cat/congressos/congres-catala-dretambiental/presentaciones>.

SHI, Y., "Reducing greenhouse gas emissions from international shipping: Is it time to consider market-based measures?”, Marine Policy, vol. 64, febrero 2016.

TAUDAL POULSEN, R., PONTE, S. y SORNN-FRIESE, H., "Environmental upgrading in global value chains: The potential and limitations of ports in the greening of maritime transport", Geoforum, vol. 89, febrero 2018.

TRIAS PRATS, B., El régimen económico de los servicios portuarios en los puertos estatales, Iustel, Madrid, 2011.

WAN, Z., MAKHLOUFI, A., CHEN, Y. y TANG, J., "Decarbonizing the international shipping industry: Solutions and policy recommendations”, Marine Pollution Bulletin, vol. 126, enero 2018. 